\title{
On triviality of the mod $p$ Hopf invariant
}

\author{
By Nobuo Shimada and Tsuneyo Yamanoshita \\ (Received Sept. 16, 1961)
}

Introduction. Recently J. F. Adams [2,3] gave an axiomatic treatment of stable secondary cohomology operations, and, applying this, proved the non-existence of elements of Hopf invariant one in $\pi_{2 n-1}\left(S^{n}\right)(n \geqq 16)$. Owing to that work, the secondary cohomology operations turn out to be a useful tool in the algebraic topology, along with the primary operations, provided with satisfactory practical machinery, which will thus serve to solve not a few interesting problems.

In the present paper, following the general scheme given by Adams, we shall extend his result on the mod 2 Hopf invariant to the $\bmod p$ case, where $p$ is an odd prime. It will be shown that the $\bmod p$ Hopf invariant

$$
H_{p}: \pi_{m+n-1}\left(S^{m}\right) \rightarrow Z_{p} \quad\left(n=2 p^{k}(p-1)\right)
$$

is trivial for $k \geqq 1$ (Theorem 5. 3); the special case $k=1$ was already obtained by Toda [17]. Our result is deduced from the following theorem: For $k \geqq 1$ the reduced power operation $\mathfrak{P}^{p^{k}}$ can be expressed in the form $\sum a_{i} \Phi_{i}$, where $\Phi_{i}$ are the basic secondary operations (\$3) and $a_{i}$ are suitable elements of the Steenrod algebra $A$ over $Z_{p}$ (Theorem 5.1 and 5.2). In choosing the basic secondary operations as generating system for secondary operations, we make use of Toda's result on exact sequences in the Steenrod algebra ([17] and Mukohda [9]) which may also be obtained by a computation of the cohomology of the Steenrod algebra [1]. Our problem, then, reduces to calculating the basic operations in the complex projective space $P$ of infinitely-many dimensions, and this will be achieved in two ways, the one making use of functional operations $(\$ 6)$ and the other making use of a formula for the composite operation $\Phi_{-1},{ }_{k} c\left(\mathfrak{P}^{p^{k(p-1)}}\right)(\S 7)$.

The contents of this paper were previously announced in [14] and [18]. The same problem of the $\bmod p$ Hopf invariant has been solved independently by A. Liulevicius [7].

The authors express their hearty thanks to Professors S. Iyanaga and A. Komatu for their encouragements during the preparation of this paper.

$\S 1$. Stable secondary cohomology operations and primary functional operations.

Let $p$ be an odd prime. We shall denote by $A$ the Steenrod algebra [6] over the prime field $Z_{p}$. As is well known, the algebra $A$ is generated by the powers $\mathfrak{P}^{i}$ and the operator $\Delta$. By $\Delta$ we mean the Bockstein operator ([6]) associated with the exact sequence of coefficients

$$
0 \rightarrow Z_{p} \stackrel{(-1)^{i} p}{\longrightarrow} Z_{p^{2}} \rightarrow Z_{p} \rightarrow 0
$$

for $i$-dimensional cohomology classes. $A$ has an additive base over $Z_{p}$ consisting of the following "admissible" monomials [5]: 


$$
\begin{aligned}
& \mathfrak{P}\left(\Delta^{\varepsilon_{0}}, i_{1}, \Delta^{\varepsilon_{1}}, \cdots \cdots, i_{r}, \Delta^{\varepsilon_{r}}\right) \text { for } \varepsilon_{j}=0 \text { or } 1, i_{j} \geqq 0 \\
& \quad \text { and } i_{j} \geqq p i_{j+1}+\varepsilon_{j} ; j=1, \cdots \cdots, r-1,
\end{aligned}
$$

where $\mathfrak{P}\left(\Delta^{\varepsilon_{0}}, i_{1}, \Delta^{\varepsilon_{1}}, \ldots \ldots, i_{r}, \Delta^{\varepsilon_{r}}\right)$ stands for $\Delta^{\varepsilon_{0}} \mathfrak{P}^{i_{1}} \ldots \ldots \mathfrak{P}^{i_{r}} \Delta^{\varepsilon_{r}}$. ( $\varepsilon_{j}$ takes values 0 or 1 and $\Delta^{0}=i d$. We omit $\Delta^{0}$ in $\mathfrak{P}\left(\Delta^{\varepsilon_{0}}, i_{1}, \Delta^{\varepsilon_{1}}, \cdots \cdots, i_{r}, \Delta^{\varepsilon_{r}}\right)$ so that, for instance $\mathfrak{P}(r, s)=\mathfrak{F}^{r} \mathfrak{F}^{s}$. This abbreviation will be used throughout the paper.) Between the generators $\mathfrak{F}^{i}$ and $\Delta$ the following relations hold ([5] and [6]):

$$
\begin{aligned}
\mathfrak{P}(r, s) & =\sum_{i \geqq 0}(-1)^{r+i}\left(\begin{array}{c}
(s-i)(p-1)-1 \\
r-i p
\end{array}\right) \mathfrak{P}(r+s-i, i) \\
& \text { for } r<p s, \\
\mathfrak{P}(r, \Delta, s) & =\sum_{i \geqq 0}(-1)^{r+i}\left(\begin{array}{c}
(s-i)(p-1) \\
r-i p
\end{array}\right) \mathfrak{P}(\Delta, r+s-i, i) \\
& +\sum_{i \geqq 0}(-1)^{r+i-1}\left(\begin{array}{c}
(s-i)(p-1)-1 \\
r-i p-1
\end{array}\right) \mathfrak{P}(r+s-i, \Delta, i) \\
\Delta \Delta & =0 .
\end{aligned}
$$

In particular [5], we have

$$
\begin{gathered}
\mathfrak{P}(r, \Delta, s)=\left(\begin{array}{c}
r+s-1 \\
r
\end{array}\right) \mathfrak{P}(\Delta, r+s)+\left(\begin{array}{c}
r+s-1 \\
s
\end{array}\right) \mathfrak{P}(r+s, \Delta) \\
\text { for } r<p .
\end{gathered}
$$

We shall recall the axioms and the fundamental properties of the stable secondary cohomology operations [2]. By an $A$-module we mean a graded left module over the graded algebra $A$. Let us write $H^{*}(X)$ for the singular cohomology ring $\sum_{q \geq 0} H^{q}\left(X, Z_{p}\right)$ of a space $X$ and write $H^{+}(X)$ for $\sum_{i} H^{q}\left(X, Z_{p}\right) ; H^{*}(X)$ and $H^{+}(X)$ are $A$-modules.

Let $C_{0}, C_{1}$ be free $A$-modules of locally finite type such that $\left(C_{i}\right)_{q}=0$ if $q<i(i=0,1)$. Let $(d, z)$ be a pair of an $A$-map $d: C_{1} \rightarrow C_{0}$ of degree zero and a $d$-cycle $z$ (i.e. a homogeneous element of Ker $d$ ). $A$ stable secondary operation $\Phi$ associated with $(d, z)$, by definition, satisfies the following axioms.

Axiom (1). $\Phi(\varepsilon)$ is defined for each $A$-map $\varepsilon: C_{0} \rightarrow H^{+}(X)$ of degree $m \geqq 1$ such that $\varepsilon d=0$.

For the next axiom, set $\operatorname{deg} z=n+1$, let $f: C_{1} \rightarrow H^{+}(X)$ run over the $A$-maps of degree $m-1$, and let $Q^{m+n}(d, z ; X)$ be the set of elements of the form $f(z)$.

Axiom (2). $\Phi(\varepsilon) \in H^{m+n}(X) / Q^{m+n}(d, z ; X)$.

Axiom (3). $g^{*} \Phi(\varepsilon)=\Phi\left(g^{*} \varepsilon\right)$ for a map $g: Y \rightarrow X$.

Let $(X, Y)$ be a pair of a space $X$ and a subspace $Y$ of $X$; let $i: Y \rightarrow X$ be the injection, and let $\varepsilon: C_{0} \rightarrow H^{+}(X)$ be an $A$-map of degree $m \geqq 1$ such that $\varepsilon d=0$ and $i^{*} \varepsilon=0$. Then we can form the following diagram.

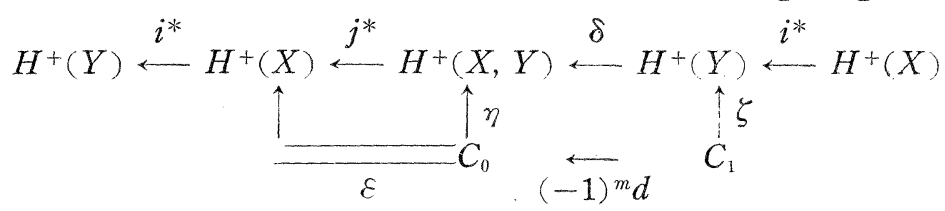


Axiom (4). $i^{*} \Phi(\varepsilon)=\{\zeta z\} \bmod i^{*} Q^{m+n}(d, z ; X)$.

For the next axiom, let $\sigma: H^{+}(X) \rightarrow H^{+}(S X)$ be the suspension isomorphism.

Axiom (5). $\quad \sigma \Phi(\varepsilon)=\Phi(\sigma \varepsilon)$.

In particular, if $C_{0}$ is free on one given generator $e_{0}$ and $\varepsilon e_{0}=u$, then we write $\Phi(u)$ for $\Phi(\varepsilon)$, and we say that $\Phi(u)$ is a stable secondary operation of one variable. A stable secondary operation of one variable is concretely defined from a relation in the Steenrod algebra as follows: Let $\sum_{a} a_{\iota} b_{t}=0\left(a_{t}, b_{t} \in A\right)$ be such a relation, let $C_{1}$ be free on symbolic generators $\left[b_{t}\right]$, and let $d: C_{1} \rightarrow C_{0}$ be an $A$-map of degree zero such that $d\left[b_{t}\right]=\mathrm{b}_{t} e_{0}$. Then we have a $d$-cycle $z=\sum_{i} a_{t}\left[b_{t}\right]$, and a stable secondary operation $\Phi$ associated with $(d, z)$ is called also associated with the relation $\sum_{t} a_{i} b_{t}=0$. In this case, the domain of definition of $\Phi$ is $\bigcap_{t} \operatorname{Ker} b_{t} \subset H^{m}(X)$ and the indeterminacy $Q^{m+n}(d, z ; X)=\sum_{t} \operatorname{Im} a_{t}$.

The following theorem of Adams [2] is basic for the present paper.

THEOREM 1.1. (1) Given $(d, z)$ as above, there is at least one stable secondary cohomology operation $\Phi$ associated with $(d, z)$.

(2) If $\Phi, \Phi^{\prime}$ are two operations associated with the same $(d, z)$ then there is an element $e$ in $\left(C_{0} ! d C_{1}\right)_{n}$ such that

$$
\Phi(\varepsilon)-\Phi^{\prime}(\varepsilon)=\{\varepsilon e\} .
$$

(3) Suppose given $d$, element $z_{t}$ in $\operatorname{Ker} d$, and operations $\Phi_{t}$ associated with the pairs $\left(d, z_{t}\right)$. Suppose $z=\sum_{t} a_{t} z_{t}\left(a_{\iota} \in A\right)$. Then there is an operation $\Phi$ associated with $(d, z)$ such that

$$
\sum_{t} a_{l} \Phi_{t}(\varepsilon)=\{\Phi(\varepsilon)\} \bmod \sum a_{i} Q^{m+n} t\left(d, z_{t} ; X\right) .
$$

(4) Suppose given a diagram

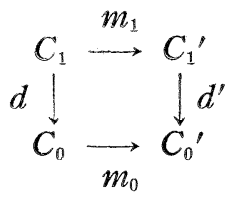

in which $d, d^{\prime}$ are as above, and $m_{0}, m_{1}$ are $A$-maps of degree zero. Let $\Phi$ be an operation associated with a pair $(d, z)$. Then there is an operation $\Phi^{\prime}$ associated with $\left(d^{\prime}, m z\right)$ such that

$$
\Phi\left(\varepsilon^{\prime} m_{0}\right)=\left\{\Phi^{\prime}\left(\varepsilon^{\prime}\right)\right\}
$$

for each $\varepsilon^{\prime}: C_{0}{ }^{\prime} \rightarrow H^{+}\left(X, Z_{p}\right)$ of the sort considered above.

As is well known, the functional primary cohomology operations are closely related to the secondary operations $[12,15]$. For later use we shall state the axioms and fundamental properties of the stable functional primary operation.

With the above notations, let $C_{0}, C_{1}$ be free $A$-modules of locally finite type such that $\left(C_{i}\right)_{q}=0$ if $q<i(i=0,1)$. Let $(d, v)$ be a pair of an $A$-map $d: C_{1} \rightarrow C_{0}$ of degree zero and a homogeneous element $v$ of $C_{1}$. We call $\phi$ a stable functional primary cohomology operation associated with $(d, v)$, if it satisfies the following axioms.

F1. $\varphi(f, \varepsilon)$ is defined for each pair of a map $f: Y \rightarrow X$ and an $A$-map $\varepsilon: C_{0} \rightarrow H^{+}(X)$ of degree $m \geqq 1$ such that $f^{*} \varepsilon=0$ aud $\varepsilon d=0$. 
In particular, if $C_{0}=A$ and $C_{1}$ is free on one generator $[a]$ and $d[a]$ $=a \in A$, then we write $u=\varepsilon(1)$ and we have $a u=0$ and $f^{*} u=0$. In this case we write $a_{f}(u)$ for $\varphi(f, \varepsilon)$ as usual, where $\varphi$ is associated with $(d,[a])$.

For the next axiom, set $\operatorname{deg}_{1}(v)=\nu$, let $\lambda: C_{0} \rightarrow H^{*}(Y), \mu: C_{1} \rightarrow H^{*}(X)$ run over the $A$-maps of degree $m-1$, and let $L^{m+\nu-1}(d, v ; f)$ be the set of elements of the form $\lambda d v+f^{*} \mu v$.

F2. $\phi(f, \varepsilon) \in H^{m+\nu-1}(Y) / L^{m+\nu-1}(d, v ; f)$.

For the next axiom, consider the following homotopy commutative diagram.

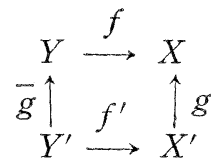

F3. $\phi\left(f^{\prime}, g^{*} \varepsilon\right)=g^{*} \phi(f, \varepsilon)$.

For the next axiom, let $(X, Y)$ be a pair, and let $\varepsilon: C_{0} \rightarrow H^{+}(Y)$ be a map of degree $m \geqq 1$ such that $\varepsilon d=0$ and $i^{*} \varepsilon=0$. We can form the same diagram as in the above Axiom (4).

F4. $\varphi(i, \varepsilon)=\{\zeta(v)\} \bmod L^{m+\nu-1}(d, v ; i)$.

F5. $\sigma \varphi(f, \varepsilon)=\varphi(S f, \sigma \varepsilon)$.

THEOREM 1.2. (1) Given a pair $(d, v)$ as above, there is a uniquely determined stable functional operation $\varphi$ associated with it (in the sense of the axioms above).

(2) Suppose given d, elements $v_{t}$ in $C_{1}$ and operations $p_{t}$ associated with the pairs $\left(d, v_{t}\right)$ respectively. Suppose $v=\sum_{t} a_{t} v_{t}\left(a_{t} \in A\right)$. Then, we have

$$
\{\phi(f, \varepsilon)\}=\left\{\sum_{t} a_{t} \boldsymbol{\varphi}_{t}(f, \varepsilon)\right\} \bmod \sum_{t} a_{t} L^{m+\nu_{t}-1}\left(d, v_{t} ; f\right)
$$

for the operation $\rho$ associated with $(d, v)$.

(3) Suppose given a diagram

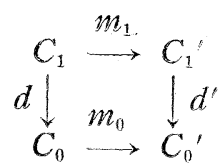

in which $d, d^{\prime}$ are as above, and $m_{0}, m_{1}$ are A-maps of degree zero. Let $q$ be the operation associated with a pair $(d, v)$, and let $\phi^{\prime}$ be the operation associated with $\left(d^{\prime}, m_{1} v\right)$. Then we have

$$
\varphi\left(f, \varepsilon^{\prime} m_{0}\right)=\left\{\mathscr{\rho}^{\prime}\left(f, \varepsilon^{\prime}\right)\right\}
$$

where $\varepsilon^{\prime}: C_{0}{ }^{\prime} \rightarrow H^{+}(X)$ is of the sort considered above.

This theorem is easily deduced from the axioms above. follows.

One may generalize the two formulas of Peterson and Stein [12] as

THEOREM 1.3. (1) Suppose given $d$ as above, element $z=\sum_{t} a_{t} e_{t}\left(a_{t} \in A\right)$ in $\operatorname{Ker} d$, where the elements $e_{t}$ form an A-base of $C_{1}$. Let $\Phi$ be any stable secondary cohomology operation associated with $(d, z)$, and let $\varphi_{t}$ be the functional operations associated with $\left(d, e_{t}\right)$. Then

$$
f^{*} \Phi(\varepsilon)=\sum_{t} a_{t} \Phi_{t}(f, \varepsilon) \bmod \sum_{t} a_{t} L^{m+\nu_{t}-1}\left(d, e_{t} ; f\right)
$$


for each $\varepsilon: C_{0} \rightarrow H^{+}(X)$ of degree $m \geqq 1$ and each $f: Y \rightarrow X$ such that $\varepsilon d=0$ and $f^{*} \varepsilon=0$.

(2) Suppose given $d$ as above, element $z$ in Ker $d$. Let $\bar{C}$ be a free A-module generated by one generator $\bar{e}$, let $\bar{d}: \bar{C} \rightarrow C_{1}$ be an A-map of degree zero such that $\bar{d}(\bar{e})=z$ and let $\bar{\rho}$ be the functional operation associated with $(\bar{d}, \bar{e})$. Then there exists a secondary operation $\Phi$ associated with $(d, z)$ such that

$$
\Phi(f * \varepsilon)=-\bar{\phi}(f, \varepsilon d) \bmod L^{m+\nu-1}(\overleftarrow{d}, \bar{e} ; f)
$$

for each $\varepsilon: C_{0} \rightarrow H^{*}(X)$ of degre $m \geqq 1$ and each $f: Y \rightarrow X$ such that $f^{*} \varepsilon d=0$.

The first formula of this theorem is nothing but a rewriting of the above Axiom (4) for secondary operations by using the axiom F4 and Theorem 1.2.

In order to prove the second formula, we use the universal examples. We begin with some preliminaries. Let $C_{0}$ be free on generators $e_{j}$ of degree $\mu_{j}$. Let $\mu=\operatorname{Min} \mu_{j}$. Let $B_{0}$ be a generalized Eilenberg-MacLane space with fundamental classes $y_{j}$ of degree $m+\mu_{j}$ (i.e. $B_{0}$ is a cartesicn product of spaces $\left.K\left(Z_{p}, m+\mu_{j}\right)\right)$. We can define an $A$-map $\theta_{0}: C_{0} \rightarrow H^{+}\left(B_{0}\right)$ of degree $m$ by $\theta_{0}\left(e_{j}\right)=y_{j}$, then $\theta_{0}$ is an isomorphism in the stable range (i.e. the range of degrees $<2(m+\mu))$. Similarly define $B_{1}, \theta_{1}$ and $\bar{B}, \bar{\theta}$ corresponding to $C_{1}$ and $\bar{C}$ ( $m$ being fixed). We can define two maps $\psi_{0}$ : $B_{0} \rightarrow B_{1}$ and $\psi_{1}: B_{1} \rightarrow \bar{B}$ such that

$$
\text { (1.4) } \psi_{0} * \theta_{1}=\theta_{0} d, \quad \psi_{1} * \bar{\theta}=\theta_{1} \bar{d}
$$

Let $\left(\Omega B_{1}, E_{0}, B_{0}\right)$ be the fibre space over base $B_{0}$ with fibre $\Omega B_{1}$ induced by the map $\psi_{0}$ from the path-space fibering $\left(\Omega B_{1}, L B_{1}, B_{1}\right)$ introduced by Serre [13]. Let $\left(\Omega \bar{B}, E_{1}, B_{1}\right)$ be similarly defined by the map $\psi_{1}$ and the fibering $(\Omega \bar{B}, L \bar{B}, \bar{B})$. Let $\sigma_{1}: H^{*}\left(B_{1}\right) \rightarrow H^{*}\left(\Omega B_{1}\right)$ and $\bar{\sigma}: H^{*}(\bar{B}) \rightarrow H^{*}(\Omega \widetilde{B})$ be the suspension homomorphisms of degree -1 . We can form the follow. ing diagram

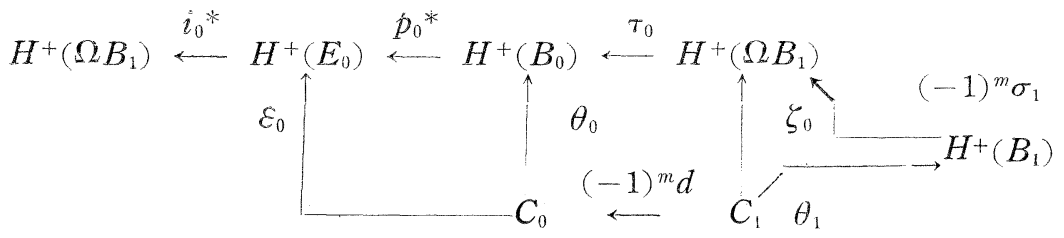

where $i_{0}: \Omega B_{1} \rightarrow E_{0}$ is the injection, $p_{0}: E_{0} \rightarrow B_{0}$ is the projection and $\tau_{0}$ is the transgression. We have a similar diagram corresponding to the fibre space $\left(\Omega \bar{B}, E_{1}, B_{1}\right)$. In particular, we may set

$$
\zeta_{0}=(-1)^{m} \sigma_{1} \theta_{1}, \quad \zeta_{1}=(-1)^{m} \bar{\sigma} \bar{\theta} \text {. }
$$

Now take $z \in \operatorname{Ker} d$ and $\bar{e} \in \bar{C}$ as in the theorem, then, from (1.4) and (1.5), we have

$$
\begin{aligned}
\zeta_{0}(z) & =\zeta_{0} \bar{d}(e)=(-1)^{m} \sigma_{1} \theta_{1} \bar{d}(\bar{e})=(-1)^{m} \sigma_{1} \psi_{1} * \bar{\theta}(\bar{c}) \\
& =(-1)^{m}\left(\Omega \psi_{1}\right)^{*} \bar{\sigma} \bar{\theta}(\bar{e})=\left(\Omega \psi_{1}\right)^{*} \zeta_{1}(\bar{e}) .
\end{aligned}
$$

Let $g: X \rightarrow B_{0}$ be a map such that 
(1.7)

$$
g * \theta_{0}=\varepsilon
$$

Then we can form the following (homotopy commutative) diagram (cf. Nomura [11])

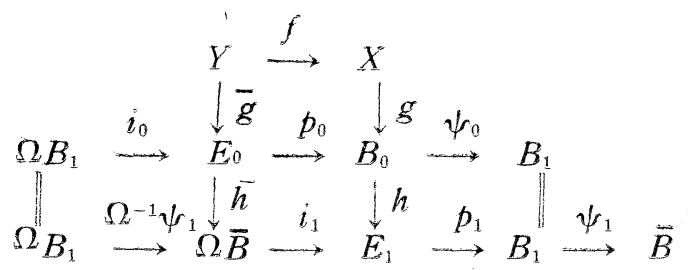

where $\Omega^{-1}$ means the composition of the loop functor and of the inversion of loops, so that we may regard $\left(\Omega^{-1} \psi_{1}\right)^{*}$ as $-\left(\Omega \psi_{1}\right)^{*}$ in the stable range.

Let $\bar{\phi}$ be the functional operation associated with $(\bar{d}, \bar{e})$. Then the axiom 4 implies

$$
\overline{\boldsymbol{\Phi}}\left(i_{1}, p_{1}^{*} \theta_{1}\right)=\left\{\zeta_{1}(\bar{e})\right\} \bmod L^{m+\nu-1}\left(\bar{d}, \bar{e} ; i_{1}\right) .
$$

Making use of the axiom F3, (1.4), (1.6) and (1.8), we have

$$
\begin{aligned}
i_{0} * \overline{\boldsymbol{\phi}}\left(p_{0}, \theta_{0} d\right)= & i_{0} * \bar{h} * \overline{\boldsymbol{\phi}}\left(i_{1}, p_{1} * \theta_{1}\right) \\
= & i_{0} * \bar{h}^{*}\left\{\zeta_{1}(\bar{e})\right\}=\left\{\left(\Omega^{-1} \psi_{1}\right) * \zeta_{1}(\bar{e})\right\}=-\left\{\zeta_{0}(\boldsymbol{z})\right\} \\
& \quad \bmod i_{0} * L^{m+\nu-1}\left(\bar{d}, \bar{e} ; p_{0}\right)
\end{aligned}
$$

where the subgroup $i_{0} * L^{m+\nu-1}\left(\bar{d}, \bar{e} ; p_{0}\right)$ is equal to $i_{0}{ }^{*} Q^{m+\nu-1}\left(d, z ; E_{0}\right)$. Therefore, from the Axiom (4), we can assert that there exists a secondary operation $\Phi$ associated with the pair $(d, z)$ such that

$$
\Phi\left(p_{0}^{*} \theta_{0}\right)=-\overline{\boldsymbol{\phi}}\left(p_{0}, \theta_{0} d\right) \bmod L^{m+\nu-1}\left(\bar{d}, \bar{e} ; p_{0}\right)
$$

It follows immediately that

$$
\begin{aligned}
\Phi(f * \varepsilon) & =\Phi\left(f^{*} g^{*} \theta_{0}\right)=\Phi\left(\bar{g}^{*} p_{0}^{*} \theta_{0}\right)=\bar{g} * \Phi\left(p_{0}^{*} \theta_{0}\right) \\
& =-\bar{g} * \overline{\boldsymbol{\rho}}\left(p_{0}, \theta_{0} d\right)=-\overline{\boldsymbol{\Phi}}(f, \varepsilon d) \bmod L^{m+\nu-1}(\bar{d}, \bar{e} ; f) .
\end{aligned}
$$

It follows from Theorem 1.1. (2) that

$$
\Phi^{\prime}\left(f^{*} \varepsilon\right)=\Phi\left(f^{*} \varepsilon\right) \quad \bmod L^{m+y-1}(\bar{d}, \bar{e} ; f)
$$

for another operation $\Phi^{\prime}$ associated with the pair $(\boldsymbol{d}, z)$. Thus we have the required result.

For later use we mention of a special case of Th. 1.3, (2).

THEOREM 1.4. Let $\sum_{t} a_{t} b_{t}=0$ be a relation in $A$, where $b_{t}$ are of positive degree. Let $f: Y \rightarrow X$ be a map. Let $u \in H^{m}(X)$ be a class such that $a_{t} b_{t} u=0$ and $f^{*} b_{t} u=0$ for any $t$. Then, for any stable secondary operation $\Phi$ associated with the relation $\sum_{t} a_{t} b_{t}=0$, we have

$$
\Phi\left(f^{*} u\right)=-\sum_{t} a_{t},{ }_{f}\left(b_{t} u\right) \quad \bmod \operatorname{Im} f^{*}+\sum_{t} \operatorname{Im} a_{t},
$$

where $a_{t}, 1$ denotes the functional operation.

\section{$\S 2$. The Steenrod algebra and the conjugation operation.}

We begin with introducing the following left and right ideals of $A$. For $k \geqq 1$, put

$$
\begin{aligned}
& M(k)=A \mathfrak{P}^{p^{k}}+\cdots \cdots+A \mathfrak{P}^{1}+A \Delta, \\
& M^{\prime}(k)=A \mathfrak{P}^{p^{k}}+\cdots \cdots+A \mathfrak{P}^{1}, \\
& M_{*}(k)=\mathfrak{P}^{p^{k}} A+\cdots \cdots \mathfrak{P}^{1} A+\Delta A, \\
& M_{*}^{\prime}(k)=\mathfrak{P}^{p^{k}} A+\cdots \cdots+\mathfrak{P}^{1} A,
\end{aligned}
$$


and $\quad M(-1)=A \Delta, \quad M_{*}(-1)=\Delta A, \quad M^{\prime}(-1)=M_{*}^{\prime}(-1)=0$,

and for $k<-1$, put

$$
M(k)=M^{\prime}(k)=M_{*}(k)=M_{*}^{\prime}(k)=0 .
$$

Furthermore, define a homomorphism $L_{k}(k \geqq-1)$ of $A$ into $A / M_{*}(k-1)$ as follows :

$$
\begin{array}{lrl}
L_{k}(a)=\mathfrak{P}^{k^{k}} a & \bmod M_{*}(k-1) & (k \geq 0) \\
L_{-1}(a)=\Delta a & \text { for } & a \in A .
\end{array}
$$

It is well-known that the kernel of $L_{-1}$ is equal to $\Delta A$.

We cite the following results of Toda ([17] and Mukohda [9]).

Proposition 2.1. For $k \geqq 1$, the kernel of $L_{k}$ is given by

$$
M_{*}(k-2)+\mathfrak{P}^{2 p^{k-1}} A+\left(2 \mathfrak{P}^{p^{k+p}+p^{k-1}}-\mathfrak{S}^{p^{k}} \mathfrak{P}^{p^{k-1}}\right) A+\mathfrak{P}^{p^{k}(p-1)} A .
$$

Proposition 2.2. The kernel of $L_{0}$ is given by

$$
\left(2 \Delta \mathfrak{P}^{1}-\mathfrak{P}^{1} \Delta\right) A+\mathfrak{P}^{p-1} A .
$$

Next, we have the following

Lemma 2.3. For $k \geqq 0,0<j<p$,

$$
\mathfrak{P}^{j p^{k}} \mathfrak{P}^{p^{k+1} l} \equiv \mathfrak{P}^{p^{k+1}+p^{k}} \bmod M^{\prime}(k-1) \cap M_{*}{ }^{\prime}(k-1) .
$$

Proof. We will proceed by induction on $k$. The assertion is trivial for $k=0$. Assume that the lemma is true for $k \leqq n-1$.

Let us verify it for $k=n$. By Adem's relation, we have

$$
\mathfrak{B}\left(j p^{n}, p^{n+1} l\right)=\mathfrak{P}\left(p^{n+1} l+j p^{n}\right)+\sum_{i=1}^{s} * \mathfrak{B}\left(p^{p+1} l+j p^{n}-i, i\right) .
$$

On the other hand, by the hypothesis of induction it is easy to see that $\mathfrak{P}\left(p^{n+1} l+j p^{n}-i, i\right)$ is contained in not only $M^{\prime}(n-1)$ but also $M_{*}^{\prime}(n-1)$ for $1 \leqq i \leqq j p^{n-1}$. q.e.d.

From now on we shall consider the conjugation operation $c$ [8] which is an anti-automorphism of the Steenrod algebra $A$ with the following properties:

$$
\begin{aligned}
& c(a b)=(-1)^{r s} c(b) c(a), \text { for } a \in A_{r}, b \in A_{s}, \\
& c(\Delta)+\Delta=0, \sum_{i \geqq 0} \mathfrak{P}^{i} c\left(\mathfrak{P}^{t-i}\right)=0 \text { for } t>0, c^{2}=\text { identity. }
\end{aligned}
$$

LEMMA 2.4. (1) For $k \geqq 0$,

$$
c\left(\mathfrak{P}^{p k}\right) \equiv-\mathfrak{P}^{p^{k}} \bmod M^{\prime}(k-2) \cap M_{*}^{\prime}(k-2),
$$

(2) For $k \geqq 0$ and $j<p$

$$
c\left(\mathfrak{P}^{3 p^{k}}\right) \equiv(-1)^{j \mathfrak{s} \mathfrak{\beta}^{j k}} \bmod M^{\prime}(k-1) \cap M_{*}{ }^{\prime}(k-1) .
$$

Proof. We shall prove this lemma by induction on $k$. From (2.1) it is easily seen that $c\left(\mathfrak{P}^{j}\right)=(-1)^{j} \mathfrak{P}^{j}$ for $j<p$. Thus the lemma is true for $k=0$. Assume that the lemma is true for $\mathrm{k} \leqq r-1$. We shall prove it for $k=r$.

First, using Lemma 2.3, we can observe that

$$
\begin{aligned}
c\left(\mathfrak{P}\left(p^{k+1} l+j p^{k}\right)\right) & \equiv c\left(\mathfrak{P}\left(j p^{r}, p^{k+1} l\right)\right) \\
& \equiv c\left(\mathfrak{P}^{p k+1}\right) c\left(\mathfrak{P}^{j} p^{k}\right) \\
& \equiv(-1)^{j} c\left(\mathfrak{P}^{p^{k+1} l}\right) \mathfrak{P}^{j p^{k}} \bmod M^{\prime}(k-1),
\end{aligned}
$$

for $k \leqq r-1$. From (2.1) and (2.2), we obtain 


$$
c\left(\mathfrak{P}^{p^{r}}\right)+\mathfrak{P}^{k^{r}}+\sum_{i=1}^{p-1} \mathfrak{P}^{i p^{r-1}} c\left(\mathfrak{P}^{(p-i) k^{r-1}}\right) \equiv 0 \bmod M^{\prime}(r-2) .
$$

On the other hand, from the Adem relation we have

$$
\mathfrak{B}^{i p^{r-1} \mathfrak{P}^{j p^{r-1}}} \equiv\left(\begin{array}{c}
i+j \\
i
\end{array}\right) \mathfrak{B}^{(i+j) p^{r-1}} \bmod M^{\prime}(\boldsymbol{r}-2)
$$

for $0 \leqq i, j<p$.

From the assumption of induction, (2.3) and (2.4), it follows that

$$
\begin{array}{rlrl}
c\left(\mathfrak{P}^{p^{r}}\right)+\mathfrak{P}^{p^{r}} & \equiv \sum(-1)^{i \mathfrak{P}^{i p r-1} \mathfrak{P}^{(p-i) p^{p-1}}} & \\
& \equiv 0 \quad \bmod M^{\prime}(\boldsymbol{r}-2) .
\end{array}
$$

On the other hand, from the assumption of induction it follows immediately that $c\left(M^{\prime}(k)\right)=M_{*}{ }^{\prime}(k)$ for $k \leqq r-1$. Thus the part (1) of the lemma has been proved.

Next, we shall prove the part (2) of the lemma by induction on $j$. Assume that the assertion is verified for $j<l$. Then from (2.1) we have

$$
\begin{aligned}
& -c\left(\mathfrak{P}^{z p^{k}}\right) \equiv \sum_{i=1}^{l-1} \mathfrak{P}^{i p^{k}} c\left(\mathfrak{S}^{(l-i) p^{k}}\right)+\mathfrak{P}^{\imath k^{k}} \bmod M^{\prime}(k-1) \\
& \equiv(-1)^{l} \sum_{i=1}^{l-1}(-1)^{i} \mathfrak{P}^{i p^{k}} \mathfrak{P}^{(l-i) p^{k}}+\mathfrak{P}^{i p^{k}} \bmod M^{\prime}(k-1) \\
& \equiv(-1)^{l}\left\{\sum_{i=1}^{l-1}(-1)^{i}\left(\begin{array}{l}
l \\
i
\end{array}\right)\right\} \mathfrak{P}^{l p^{k}}+\mathfrak{P}^{l p^{k}} \bmod M^{\prime}(k-1) \\
& \equiv-(-1)^{\imath x^{3} l^{l k}} \quad \bmod M^{\prime}(k-1) \text {. }
\end{aligned}
$$

By taking the conjugates on both sides, we obtain the part (2) of the lemma. q.e.d.

Furthermore we have

LemMa 2.5. For $k \geqq 1$,

$$
c\left(\mathfrak{P}^{x^{k}+p^{k-1}}\right)=\mathfrak{P}^{p^{k}} \mathfrak{P}^{p^{k-1}} \quad \bmod M^{\prime}(k-2) .
$$

Proof: Similarly as in Proof of Lemma 2.4, we have

$$
\begin{aligned}
c\left(\mathfrak{P}^{p^{k}+p^{k-1}}\right) & +\mathfrak{F}^{p^{k-1}} c\left(\mathfrak{P}^{p^{k}}\right)+\sum_{j=2}^{n-1} \mathfrak{P}^{(p-j+1) p^{k-1}} c\left(\mathfrak{P}^{j p^{k-1}}\right) \\
& +\mathfrak{P}^{k^{k}} c\left(\mathfrak{B}^{p^{k-1}}\right)+\mathfrak{P}^{p^{k}+p^{k-1}} \equiv 0 \quad \bmod M^{\prime}(k-2) .
\end{aligned}
$$

The second term is equal to $-\mathfrak{S}^{p^{k}+p^{k-1}} \bmod M^{\prime}(k-2)$ by Lemmas 2.4 and 2.3. The third term is contained in $M^{\prime}(k-2)$ by Lemma 2.4 and (2.4). As to the fourth term, $\mathfrak{F}^{p^{k}} c\left(\mathfrak{B}^{p k-1}\right) \equiv-\mathfrak{P}^{p^{k}} \mathfrak{P}^{p^{k-1}} \bmod M^{\prime}(k-2)$. The proof is thus completed.

Remark 2.6. In case $p=2$, set $M(k)=A \mathrm{Sq}^{2 k}+\cdots \cdots+A \mathrm{Sq}^{1}, \quad M_{*}(k)=\mathrm{Sq}^{2 k} A$ $+\cdots \cdots+\mathrm{Sq}^{1} A$ and $M(k)=M_{*}(k)=0$ for $k<0$. Then we have similar relations as in the above lemmas:

$$
\begin{aligned}
& \text { For } \quad k \geqq 0, c\left(\mathrm{Sq}^{2^{2}}\right) \equiv \mathrm{Sq}^{2^{k}} \quad \bmod M(k-2) \cap M_{*}(k-2) \text {, } \\
& \text { and for } k \geqq 1, c\left(\mathrm{Sq}^{2^{k}+2^{k-1}}\right) \equiv \mathrm{Sq}^{2^{k}} \mathrm{Sq}^{2 k-1} \quad \bmod M(k-2) \text {. }
\end{aligned}
$$

\section{$\S 3$. The stable secondary cohomology operations defined for $\bigcap_{i=0}^{k} \operatorname{Ker} \mathfrak{P}^{p^{i}} \cap \operatorname{Ker} \Delta$.}

Let $C_{1}^{k}(k \geqq 0)$ be an $A$-free module with the symbolic base consisting of 
$\left[c \mathfrak{F}^{p^{i}}\right](i=0, \cdots, k)$ and $[c \Delta]$. Let $C_{1}^{-1}$ be an $A$-free module with the single base $[c \Delta]$. Then we obtain the following inclusion relation

$$
C_{1}^{k} \supset C_{1}^{k-1} \supset \cdots \cdots \supset C_{1}^{0} \supset C_{1}^{-1} .
$$

Furthermore, define a graduation for the $A$-free base as follows: deg. of $\left[\mathfrak{c P}^{p^{i}}\right]=2 p^{i}(p-1)$, deg. of $[c \Delta]=1$. This graduation can be extended to all $C_{1}^{k}$ naturally.

Now we fix one $k(-1 \leqq k)$ and substitute by $C_{1}^{k}$ and $A$ the modules $C_{1}$ and $C_{0}$ considered in $\S 1$, and we define $A$-map $d$ of $C_{1}$ into $C_{0}$ by $d\left[c \mathfrak{P}^{p^{i}}\right]$ $=c\left(\mathfrak{P}^{p^{i}}\right)(i=0,1, \cdots \cdots, k), d[c \Delta]=c(\Delta)$. Choose a homogeneous $d$-cycle $z$ of $C_{1}^{k}$, then $(d, z)$ yields a stable secondary cohomology operation $\Phi$. The domain of this $\Phi$ is given by the first formula of Lemma 3.1.

LEMMA 3.1. We have

$$
\begin{aligned}
& \bigcap_{i=0}^{k} \operatorname{Ker} c\left(\mathfrak{P}^{p^{i}}\right) \cap \operatorname{Ker} c(\Delta)=\bigcap_{i=0}^{k} \operatorname{Ker} \mathfrak{P}^{p^{i}} \cap \operatorname{Ker} \Delta, \\
& \operatorname{Im} c\left(\mathfrak{P}^{1}\right)+\cdots \cdots+\operatorname{Im} c\left(\mathfrak{P}^{p^{k}}\right)+\operatorname{Im} c(\Delta)=\operatorname{Im} \mathfrak{P}^{1}+\cdots \cdots+\operatorname{Im} \mathfrak{P}^{p^{k}}+\operatorname{Im} \Delta .
\end{aligned}
$$

This is obviously deduced from Lemma 2.4.

Next, we shall specify some $d$-cycles in the following way.

(3.1) For $k \geqq 1$,

$$
\begin{aligned}
& Z_{i, k}=c\left(\mathfrak{P}^{p^{i}}\right)\left[c \mathfrak{P}^{p^{k}}\right]+\sigma_{i, k} \quad(0 \leqq i \leqq k-2) \\
& Z_{-1, k}=c(\Delta)\left[c \mathfrak{P}^{p^{k}}\right]-c\left(\Delta \mathfrak{P}^{p^{k}-1}\right)\left[c \mathfrak{P}^{1}\right]-c\left(\mathfrak{P}^{x^{k}}\right)[c \Delta], \\
& U_{k}=c\left(\mathfrak{P}^{2 p^{k-1}}\right)\left[c \mathfrak{P}^{p^{k}}\right]+\sigma_{k}^{\prime}, \\
& V_{k}=c\left(2 \mathfrak{P}^{p^{k}+p^{k-1}}-\mathfrak{P}\left(p^{k}, p^{k-1}\right)\right)\left[c \mathfrak{P}^{p^{k}}\right]+\sigma_{k}^{\prime \prime}, \\
& W_{k}=c\left(\mathfrak{P}^{p^{k}(p-1)}\right)\left[c \mathfrak{P}^{y^{k}}\right]+\sigma_{k}^{\prime \prime \prime},
\end{aligned}
$$

(3.2) for $k=0$,

$$
\begin{aligned}
& V_{0}=c\left(2 \Delta \mathfrak{B}^{1}-\mathfrak{P}^{1} \Delta\right)\left[c \mathfrak{P}^{1}\right]-2 c\left(\mathfrak{P}^{2}\right)[c \Delta] \\
& W_{0}=c\left(\mathfrak{P}^{p-1}\right)\left[c \mathfrak{B}^{1}\right],
\end{aligned}
$$

(3.3) for $k=-1$

$$
\mathbb{Z}_{-1,-1}=c(\Delta)[c \Delta]
$$

where $\sigma_{1, k}, \sigma_{k}^{\prime}, \sigma_{k}^{\prime \prime}$ and $\sigma_{k}^{\prime \prime \prime}$ are elements of $C_{1}^{k-1}$. By a suitable choice of these elements, the elements in $(3.1),(3.2),(3.3)$ can be regarded as $d$ cycles. This is assured by Proposition 2.1 and 2.2. For example,

$$
\begin{aligned}
\alpha W_{k} & =c\left(\mathfrak{P}^{p^{k}(p-1)}\right) c\left(\mathfrak{P}^{p^{k}}\right)+d \sigma_{k}^{\prime \prime \prime} \\
& =c\left(\mathfrak{P}^{p^{k}} \mathfrak{P}^{p^{k}(p-1)}\right)+d \sigma_{k}^{\prime \prime \prime} \\
& =c\left(\mathfrak{P}^{p^{k}} \mathfrak{P}^{p^{k}(p-1)}+c^{-1}\left(d \sigma_{k}^{\prime \prime \prime}\right)\right) .
\end{aligned}
$$

By Proposition $2.1 \mathfrak{P}^{p^{k}} \mathfrak{P}^{p^{k}(p-1)}$ is contained in $M_{*}(k-1)$. On the other hand, we have $d C_{1}^{k-1}=M(k-1)$, i.e. $c^{-1}\left(d C_{1}^{k-1}\right)=M_{*}(k-1)$.

In the sequel, $\sigma_{i, k}, \sigma_{k}^{\prime}, \sigma_{k}^{\prime \prime}$ and $\sigma_{k}^{\prime \prime \prime}$ will be kept fixed. Then the stable secondary operation associated with $Z_{i, k}(0 \leqq i \leqq k-2$ or $i=-1, k=-1), U_{j}$ $(j \geqq 1), V_{k}, W_{k}(k \geqq 0)$ are uniquely determined, as $A / d C_{1}^{k}$ is zero in degrees $i, 0<i<2 p^{k}(p-1)$ (Cf. Theorem 1.1 (2)). These operations associated will be denoted by $\Phi_{i, k}, \Phi_{j}^{(0)}, \Phi_{k}^{(1)}, \Phi_{k}^{(2)}$ respectively, and will be called basic operations.

The following proposition justifies the word "basic". 
Proposition 3.2. The kernel of $d: C_{1}^{k} \rightarrow A$ is just

$$
\sum_{j \leqq k} A Z_{i, j}+\sum_{j \leqq k} A U_{j}+\sum_{j \leqq k} A V_{j}+\sum_{j \leqq k} A W_{j}
$$

Proof. We proceed by induction on. Let $k=-1$. Let $z$ be a $d$-cycle of $C_{1}^{-1}=A[c \Delta]$, which we denote by $c(\alpha)[c \Delta]$. Then we have

$$
d z=c(\alpha) c(\Delta)=c(\Delta \alpha)=0 \text {. }
$$

That is, $\Delta \alpha=0$.

As the kernel of $L_{-1}$ is equal to $\Delta A$, there exists an element $\alpha_{-1,-1}$ of $A$ such that $\alpha=\Delta \alpha_{-1},-1$. Therefore we obtain $z=c(\alpha)[c \Delta]=c\left(\alpha_{-1},-1\right) c(\Delta)[c \Delta]$ $=c\left(\alpha_{-1},-1\right) z_{-1,-1}$.

Let us assume that our assertion is verified for $r(<k)$. Now, we take a $d$-cycle $z$ of $C_{1}^{k}$, and write it in the following from

$$
z=c\left(\alpha_{k}\right)\left[c \mathfrak{P}^{z^{k}}\right]+\sum_{i=0}^{k-1} c\left(\alpha_{i}\right)\left[c \mathfrak{P}^{p^{i}}\right]+c\left(\alpha_{-1}\right)[c \Delta] .
$$

Then we have

$$
\begin{aligned}
d z & =c\left(\alpha_{k}\right) c\left(\mathfrak{P}^{p k}\right)+\sum_{i=0}^{k-1} c\left(\alpha_{i}\right) c\left(\mathfrak{P}^{p^{i}}\right)+c\left(\alpha_{-1}\right) c(\Delta) \\
& =c\left\{\mathfrak{P}^{k^{k}} \alpha_{k}+\sum_{i=0}^{k-1} \mathfrak{P}^{p^{i}} \alpha_{i}+\Delta \alpha_{-1}\right\} \\
& =0 .
\end{aligned}
$$

Thus $L_{k} \alpha_{k}=\mathfrak{P}^{k^{k}} \alpha_{k}=-\sum_{i=6}^{k-1} \mathfrak{P}^{p^{i}} \alpha_{i}-\Delta \alpha_{-1} \equiv 0$.

By Proposition 2.1 and 2.2 there exist elements $\alpha_{i, k}, \alpha_{k}^{\prime}, \alpha_{k}^{\prime \prime}, \alpha_{k}^{\prime \prime \prime}$ of $A$ such that

$$
\begin{aligned}
& \alpha_{k}=\sum_{i=0}^{k-2} \mathfrak{F}^{p^{i}} \alpha_{i_{3} k}+\Delta \alpha_{-1}, k+\mathfrak{F}^{2 p^{k-1}} \alpha_{k}^{\prime}+\left(2 \mathfrak{F}^{p^{k}+p^{k-1}}-\mathfrak{F}\left(p^{k}, p^{k-1}\right)\right) \alpha_{k}^{\prime \prime} \\
& +\mathfrak{P}^{z^{k}(p-1)} \alpha_{k}^{\prime \prime \prime} \quad(k \geqq 1), \\
& \alpha_{k}=\left(2 \Delta \mathfrak{P}^{1}-\mathfrak{P}^{1} \Delta\right) \alpha_{0}^{\prime \prime}+\mathfrak{P}^{p-1} \alpha_{0}^{\prime \prime \prime} \quad(k=0) \text {. }
\end{aligned}
$$

Therefore we obtain

$$
\begin{aligned}
z= & \left\{\sum_{i=0}^{k-1} c\left(\alpha_{1}, k\right) c\left(\mathfrak{P}^{p^{i}}\right)+c\left(\alpha_{-1}, k\right) c(\Delta)+c\left(\alpha_{k}^{\prime}\right) c\left(\mathfrak{P}^{2} p^{k-1}\right)\right. \\
& \left.+c\left(\alpha_{k}^{\prime \prime}\right) c\left(2 \mathfrak{P}^{p^{k}+p k-1}-\mathfrak{P}\left(p^{k}, p^{k-1}\right)\right)+c\left(\alpha_{k}^{\prime \prime \prime}\right) c\left(\mathfrak{P}^{p^{k}(p-1)}\right)\right\}\left[c \mathfrak{P}^{p k}\right] \\
& +\sum_{i=0}^{k-1} c\left(\alpha_{i}\right)\left[c \mathfrak{P}^{p i}\right]+c\left(\alpha_{-1}\right) c(\Delta) \quad(k \geqq 1), \\
z= & \left\{c\left(\alpha_{0}^{\prime \prime}\right) c\left(2 \Delta \mathfrak{P}^{1}-\mathfrak{P}^{1} \Delta\right)+c\left(\alpha_{0}^{\prime \prime \prime}\right) c\left(\mathfrak{P}^{p-1}\right)\right\}\left[c \mathfrak{P}^{1}\right]+c\left(\alpha_{-1}\right)[c \Delta] \quad(k=0) .
\end{aligned}
$$

Then the cycles

$$
\begin{array}{ll}
z-\sum_{i=-1}^{k-1} c\left(\alpha_{i}, k\right) Z_{i},{ }_{k}-c\left(\alpha_{k}^{\prime}\right) U_{k}-c\left(\alpha_{k}^{\prime \prime}\right) V_{k}-c\left(\alpha_{k}^{\prime \prime \prime}\right) W_{k} & \quad \text { (for } k \geqq 1), \\
z-c\left(\alpha_{0}^{\prime \prime}\right) V_{0}-c\left(\alpha_{0}^{\prime \prime \prime}\right) W_{0} & \text { (for } k=0),
\end{array}
$$

are contained in $C_{1}^{k-1}$. By the assumption of induction, the proof is completed. 
$\$ 4$. Calculation of the basic secondary operations in the complex projective space of infinitely-many dimensions.

Let us denote by $P$ the complex projective space of infinitely many dimensions and by $y$ a generator of $H^{2}\left(P, Z_{p}\right)$. Then $H^{*}\left(P, Z_{p}\right)$ is regarded as the polynomial algebra with a single generator $y$. We shall consider the effects of our basic stable secondary cohomology operations in $H^{*}\left(P, Z_{p}\right)$.

LEMMA 4.1. We have for $s>0$

$$
\begin{aligned}
& \Delta y^{s}=0 \\
& \mathfrak{P}^{r} y^{s}=\left(\begin{array}{l}
s \\
r
\end{array}\right) y^{s+r(p-1)}
\end{aligned}
$$

Since the proof is easy by induction on $s$, so it is omitted.

LEMMA 4.2. If $y^{s}$ is contained in $\cap_{i=0}^{k} \operatorname{Ker} \mathfrak{P}^{p^{i}} \cap \operatorname{Ker} \Delta$, then $s$ is a multiple of $p^{k+1}$.

This follows immediately from the computation of binomial coefficient modulo $p$ (Cf. [6]).

Thus, by Lemma 3.1 the basic operations $\Phi_{i, k}(-1 \leqq i \leqq k-2), \Phi_{k}^{(j)}(j=0$, $1,2)$ are all defined for cohomology classes of the form $y^{p k+1}$. These operations except for $\Phi_{-1, k}(k \geqq 1)$ and $\Phi_{\delta}^{(1)}$ are of odd degrees i.e. they map subgroups of $H^{n}\left(P, Z_{p}\right)$ into $H^{n+i}\left(P, Z_{p}\right), Q$ for certain subgroup $Q$ where $i$ is odd. Therefore these operations operate trivially in $H^{*}\left(P, Z_{p}\right)$.

Now, we shall show that $\Phi_{-1},{ }_{k}\left(y^{p^{k+1} n}\right)(k \geqq 1)$ and $\Phi_{0}^{(1)}\left(y^{p_{n}}\right)$ may be also calculated with null indeterminacy. $\Phi_{0}^{(1)}$ is evaluated modulo:

(4.1)

$\operatorname{Im} c\left(2 \Delta \mathfrak{P}^{1}-\mathfrak{P}^{1} \Delta\right)+\operatorname{Im} c\left(\mathfrak{P}^{2}\right)$

Obviously, the first term in (4.1) is zero as $H^{2 j+1}\left(P, Z_{p}\right)=0$. With respect to the second term, we can assert that it is zero by $c\left(\mathfrak{F}^{2}\right) y^{p_{n}}=\mathfrak{P}^{2} y^{p_{n}}=0$. Next, recall $\Phi_{-1, k}(k \geqslant 1)$ is evaluated modulo

$$
\operatorname{Im} c(\Delta)+\operatorname{Im} c\left(\Delta \mathfrak{p}^{\gamma^{k-1}}\right)+\operatorname{Im} c\left(\mathfrak{P}^{p k}\right) \text {. }
$$

Both the first and the second term are zero as $H^{2 j+1}\left(P, Z_{p}\right)=0$. As for the third term we obtain $\operatorname{Im} c\left(\mathfrak{P}^{p^{k}}\right)=0$ by $c\left(\mathfrak{P}^{x^{k}}\right) y^{p k+1_{n}}=-\mathfrak{P}^{y^{k}} y^{p^{k+1} n}=0$.

Later, in $\$ 6$ and $\$ 7$ we shall prove the following theorems.

THEOREM $4.3_{0}$. $\Phi_{0}^{(1)} y^{p n}=-n y^{p n+2(p-1)}$ mod zero.

TEFOREM 4. $3_{k} . \Phi_{-1},{ }_{k} y^{p k+1} n=-n y^{p^{k}\left(p_{n+p-1}\right)} \bmod$ zero.

\section{\$5. The mod p Hopf invariant.}

If we assume Theorem $4.3_{0}$ and Theorem $4.3_{k}$, then we can obtain the following theorems.

Theorem 5.1. For $u \in \operatorname{Ker} \mathfrak{B}^{1} \cap \operatorname{Ker} \Delta$, we have

$$
\mathfrak{P}^{p} u=\left(-\Delta \Phi_{0}^{(2)}-\mathfrak{P}^{p-2} \Phi_{0}^{(1)}\right) u \text {. }
$$

THEOREM 5.2. $\mathfrak{B}^{p+1}(k \geqq 1)$ may be expressed by stable secondary cohomology operations as follows:

$$
\begin{gathered}
\mathfrak{P}^{p^{2}} u \equiv\left(c(\Delta) \Phi_{1}^{(2)}+c\left(\Delta \mathfrak{P}^{p(p-1)-2}\right) \Phi_{1}^{(0)}-c\left(\mathfrak{P}^{p(p-1)}\right) \Phi_{-1}, 1\right) u \\
\bmod \left(A \Phi_{0}^{(1)}+A \Phi_{0}^{(2)}+A \Phi_{-1,-1}\right) u \quad(k=1)
\end{gathered}
$$




$$
\begin{aligned}
\mathfrak{B}^{p^{k-1}} u \equiv & \left(c(\Delta) \Phi_{k}^{(2)}-c\left(\Delta \mathfrak{P}^{p^{k}(p-1)-1}\right) \Phi_{0}, k-c\left(\mathfrak{P}^{p^{k}(p-1)} \Phi_{-1, k}\right) u\right. \\
& \bmod \left(\sum_{j<k} A \Phi_{i, j}+\sum_{j \gtrless k} A \Phi_{j}^{(i)}\right) u \quad(k \geq 2),
\end{aligned}
$$

where $u \in \mathrm{n}_{i=u}^{k} \operatorname{Ker} \mathfrak{F}^{p^{i}} \cap \operatorname{Ker} \Delta$.

Proof of Theorem 5.1. By the Adem relation we have

$$
\mathfrak{P}^{p-1} \Delta-\left(2 \Delta \mathfrak{P}^{1}-\mathfrak{P}^{1} \Delta\right) \mathfrak{P}^{p-2}=0 .
$$

Consequently

$$
c(\Delta) c\left(\mathfrak{P}^{p-1}\right)\left[c \mathfrak{P}^{1}\right]+c\left(\mathfrak{P}^{p-2}\right) c\left(2 \Delta \mathfrak{P}^{1}-\mathfrak{P}^{1} \Delta\right)\left[c \mathfrak{P}^{1}\right]=0 .
$$

This implies

$$
0=-\Delta W_{0}-\mathfrak{B}^{p-2} V_{0}
$$

that is,

$$
\mu_{0} \mathfrak{F}^{p}=-\Delta \Phi_{0}^{(2)}-\mathfrak{F}^{p-2} \Phi_{0}^{(1)} .
$$

If we let it operate on $y^{p}$, then by Theorem $4.3_{0}$ we have

$$
\begin{aligned}
\mu_{0} \mathfrak{P}^{p} y^{p} & =\mu_{0} y^{p^{2}} \\
& =-\mathfrak{F}^{p-2} \Phi_{0}^{(1)} y^{p}=\mathfrak{P}^{p-2} y^{p+2(p-1)}=y^{p^{2}} \text { mod zero. }
\end{aligned}
$$

Therefore we obtain $\mu_{0}=1$.

Proof of Theorem 5.2. We shall try to express $\mathfrak{P}^{p^{2}}$ by the stable secondary operations. By the Adem relation we have

$$
c\left(\Delta \mathfrak{P}^{p(p-1)}\right)+c\left(\mathfrak{P}^{2} \Delta \mathfrak{P}^{p(p-1)-2}\right)-c\left(\Delta \mathfrak{B}^{p(p-1)}\right)=0 .
$$

Therefore it holds that

$$
\left\{c(\Delta) c\left(\mathfrak{P}^{p(p-1)}\right)+c\left(\Delta \mathfrak{P}^{p(p-1)-2}\right) c\left(\mathfrak{P}^{2}\right)-c\left(\mathfrak{P}^{p(p-1)}\right) c(\Delta)\right\}\left[c \mathfrak{P}^{p}\right]=0 .
$$

This implies

$$
c(\Delta) W_{1}+c\left(\Delta \mathfrak{P}^{p(p-1)-2}\right) U_{1}-c\left(\mathfrak{P}^{p(p-1}\right) Z_{-1,1} \equiv 0 \bmod A V_{0}+A W_{0}+A Z_{-1,-1} .
$$

In other words, this asserts that the following equality holds.

$$
\begin{gathered}
\mu_{1} \mathfrak{\beta}^{p^{2}} \equiv c(\Delta) \Phi_{1}^{(2)}+c\left(\Delta \mathfrak{F}^{p(p-1)-2}\right) \Phi_{1}^{(0)}-c\left(\mathfrak{P}^{p(p-1)}\right) \Phi_{-1}, 1 \\
\bmod A \Phi_{0}^{(1)}+\Phi_{0}^{(2)}+A \Phi_{-1,-1} .
\end{gathered}
$$

Let this operate on $y^{p^{2}(p-1)}$, then by Theorem $4.3_{0}$, Theorem $4.3_{k}(k=1)$ and Lemma 2.4 we have

$$
\begin{aligned}
\mu_{1} \mathfrak{F}^{p^{2}} y^{p^{2}(p-1)} & =-\mu_{1} y^{2 p^{2}(p-1)} \\
= & -c\left(\mathfrak{P}^{p(p-1)}\right) \Phi_{-1},{ }_{1} y^{p^{2}(p-1)}=-c\left(\mathfrak{P}^{p(p-1)}\right) y^{p(p-1)(p+1)} \\
= & -y^{2 p^{2}(p-1)} \quad \bmod \text { zero. }
\end{aligned}
$$

Therefore we obtain $\mu_{1}=1$.

Let Theorem $4.3_{j}$ be proved for $j<k(\geqq 2)$ and

$$
\Phi_{-1, k} y^{p k+1(p-1)}=y^{p^{k}(p-1)(p+1)}
$$

be proved, and notice that we have then for $j \leqq k-1$

$$
\Phi_{-1, j} y^{p k+1_{n}}=0, \quad \Phi_{0}^{(1)} y^{p^{k+1} n}=0
$$

without any indeterminacy.

We shall utilize the Adem relation

$$
c\left(\mathfrak{P}^{p^{k}(p-1)} \Delta\right)-c\left(\mathfrak{P}^{1} \Delta \mathfrak{P}^{p^{k}(p-1)-1}\right)-c\left(\Delta \mathfrak{P}^{k_{(p-1)}}\right)=0
$$


corresponding to (5.2). In a similar way as above, we may, using Theorem 1. 1 , put for $k \geqq 2$

$$
\begin{aligned}
& \mu_{k} \mathfrak{B}^{p^{k+1}} \equiv c(\Delta) \Phi_{k}^{(2)}-c\left(\Delta \beta^{\left.p^{k(p-1)-1}\right)} \Phi_{0, k}-c\left(\Re^{p^{k}(p-1)}\right) \Phi_{-1, k}\right. \\
& \bmod \sum_{j<k} A \Phi_{i, j}+\sum_{j<k} A \Phi_{j}^{(i)} .
\end{aligned}
$$

Let this operate on $y^{p k+1(p-1)}$, then by (5.5), (5.4) and Lemma 2.7 we have

$$
\begin{aligned}
\mu_{k} \mathfrak{S}^{p^{k+1}} y^{p^{k+1}(p-1)}=-\mu_{k} y^{p^{k+1}(p-1)} \\
=-c\left(\mathfrak{P}^{x^{k}(p-1)}\right) \Phi_{-1, k} y^{p^{k+1}(p-1)}=-c\left(\mathfrak{P}^{p^{k}(p-1)}\right) y^{p^{k}(p-1)(p+1)} \\
=-y^{2 p^{k+1}(p-1)} \bmod \text { zero. }
\end{aligned}
$$

Consequently we obtain $\mu_{k}=1$. (q.e.d.)

Let $K=S^{m} \cup_{f} e^{m+n}(n=2 t(p-1))$ be a cell complex in which $e^{m+n}$ is attached to $S^{m}$ by a mapping $f: S^{m+n-1} \rightarrow S^{m}$. Then the following statements are equivalent :

(a) The Steenrod reduced power

$$
\text { s.t }^{t}: H^{m}\left(K, Z_{p}\right) \rightarrow H^{m+n}\left(K, Z_{p} \quad(n=2 t(p-1))\right.
$$

is zero for any mapping $f$ and any $t\left(p^{k+1}>t \geqq p^{k}\right)$

(b) For $t=p^{k}$, the above reduced power is zero.

(c) The mod $p$ Hopf invariant (homomorphism) [17]

$$
H_{p}: \pi_{m+n-1}\left(S^{m}\right) \rightarrow Z_{p} \quad\left(n=2 p^{k}(p-1)\right)
$$

is trivial.

It is a classical result that the conclusions are false for $k=0$. Here, we shall show the following theorem.

THEOREM 5.3. The mod p Hopf invariant (homomorphism)

$$
H_{p}: \pi_{m+2 p^{k}}^{k}(p-1)-1\left(S^{m}\right) \rightarrow Z_{p}
$$

is trivial for $k \geqq 1$.

Proof. Let $u$ be a non-zero element of $H^{m}\left(K, Z_{p}\right)$. Then we have $\Delta u=\mathfrak{P}^{p^{i}} u=0(i<k)$. We see by Theorem 5.1 and Theorem 5.2

$$
\mathfrak{P}^{p^{k}} u=\sum a_{i} \Phi_{i} u, \bmod a_{i} Q_{i}(u),
$$

where $\Phi_{i}$ are the secondary cohomology operations and $a_{i}$ are the elements of the Steenrod algebra with positive degrees. Obviously, we have by the reason on dimension that $\Phi_{i} u=0$, mod zero. That is, $\mathfrak{B}^{p^{k}} u=0, \bmod$ zero.

Remark 5.4. If $p=2$, we have analogues to Theorem 5.1 and Theorem 5.2 for $k \geqq 3$.

$$
\mathrm{Sq}^{2^{k+1}}=c\left(\mathrm{Sq}^{1}\right) \Phi_{k, k}+c\left(\mathrm{Sq}^{2^{k}-1}\right) \Phi_{1, k}+c\left(\mathrm{Sq}^{2^{2 k}}\right) \Phi_{0, k}+\sum_{j \leqq k=1} a_{i, j} \Phi_{i, j},
$$

where $\Phi_{i, j}$ are the stable secondary cohomology operations defined in Remark 7.4 and $a_{i, j}$ are elements of the Steenrod algebra over $Z_{2}$. From this, we can obtain Adams' theorem on the Hopf invariant [2,3].

\section{\$6. First proof.}

In this section we shall give a proof of Theorem $4.3_{k}$ by making use of Theorem 1.4. In applying Theorem 1.4, we use the complex projective space $P$ of infinitely-many dimensions as the space $Y$, a certain fibre space $\widetilde{K}_{k}$ (for each $k \geqq 0$ ) as the space $X$, and the composition $f_{k}=g_{k} \circ d$ of the 
diagonal map $d: P \rightarrow P^{p}$ (cartesian product of $p$ copies of $P$ ) and of a suitable map $g_{k}: P^{p} \rightarrow \widetilde{K}_{k}$ as the map $f: Y \rightarrow X$.

We shall begin with some preparations.

Let $p$ be an odd prime, and let $n$ be a positive integer. For each $k \geqq 0$, set $N_{k}=2 p^{k+1} n$. Then the space $\tilde{K}_{k}$ will be defined as a fibre space over the base $B_{k}=K\left(Z, N_{k}\right)$ with fibre $F_{k}$, a generalized Eilenberg-MacLane space of the following type:

$$
\begin{gathered}
F_{0}=K\left(Z, N_{0}+2(p-1)\right) \times K\left(Z, N_{0}+4(p-1)\right), \\
F_{k}=K\left(Z, N_{k}+2 p^{k}(p-1)\right) \times{\underset{j=0}{k-1} K\left(Z, N_{k}+2 p^{j}(p-1)-1\right)}_{\quad(\text { for } k \geqq 1) .}
\end{gathered}
$$

Let

$$
\begin{aligned}
& \zeta_{j} \in H^{N_{0}+2 j(p-1)}\left(F_{0}, Z\right)(j=1,2), \quad \eta_{k} \in H^{N_{k}+2 p k(p-1)}\left(F_{k}, Z\right) \\
& \qquad(k \geqq 1), \\
& \eta_{k},{ }_{j} \in H^{N_{k}+2 p^{j}(p-1)-1}\left(F_{k}, Z_{p}\right)(k \geqq 1,0 \leqq j \leqq k-1) \text { and } \\
& \iota_{k} \in H^{N_{k}}\left(B_{k}, Z\right)(k \geqq 0) \text { be fundamental classes of the respective }
\end{aligned}
$$

spaces.

Denote by $\tau$ the transgression homomorphism. Then the fibre space $\left(F_{k}, \widetilde{K}_{k}, B_{k}\right)$ is to be defined so that

$$
\begin{aligned}
& \tau \zeta_{j}=\Delta * \mathfrak{\beta}^{j}\left(\iota_{0}\right)_{p} \text { for } k=0, \\
& \tau \eta_{k}=\Delta * \mathfrak{F}^{*^{k}}\left(\iota_{k}\right)_{p}, \\
& \tau \eta_{k}, j=\mathfrak{F}^{j}\left(\iota_{k}\right)_{p} \quad(0 \leqq j \leqq k-1) \text { for } k \geqq 1,
\end{aligned}
$$

where $\Delta^{*}$ denotes the Bockstein operator associated with the exact sequence

$$
0 \rightarrow Z \stackrel{(-1)^{i} p}{\longrightarrow} Z \longrightarrow Z_{p} \longrightarrow 0
$$

and ()$_{p}$ denotes the reduction $\bmod p$ of coefficients.

The following lemma is well known.

Lemma 6.1. Let $f: Y \rightarrow X$ be a map, and let $v \in H^{m}(X, Z) \eta \in H^{m}(Y, Z)$ be such classes that $f^{*} v=p \eta$. Then we have

$$
\Delta_{t}(v)_{p}=\{\eta p\} \bmod \operatorname{Im} f^{*}+\operatorname{Im} \Delta .
$$

For the following lemmas, let $i: F_{k} \rightarrow \widetilde{K}_{k}$ be the injection, $\rho: \widetilde{K}_{k} \rightarrow B_{k}$ the projection, and set $\bar{\iota}_{k}=\rho^{*} \iota_{k}$.

Lemma 6. 20. There exist classes $v_{0}, j \in H^{N_{0}+2 j(p-1)}\left(\tilde{K}_{0}, Z\right)(j=1,2)$ such that

$$
\begin{aligned}
& i^{*} v_{0}, j=p \zeta_{j}, \\
& \left(v_{0}, j\right) p=\mathfrak{F}^{j}\left(\bar{\iota}_{0}\right)_{p} \quad(j=1,2) .
\end{aligned}
$$

Lemma $6.2_{k}$. For each $k \geqq 1$, there exists a class $v_{k} \in H^{\mathbb{N}_{k}+2 p k_{(p-1)}}\left(\tilde{K}_{k}, Z\right)$ such that

$$
\begin{aligned}
& i^{*} v_{k}=p \eta_{k}, \\
& \left(v_{k}\right)_{p}=\mathfrak{P}^{z^{k}}\left(\bar{\iota}_{k}\right)_{p} .
\end{aligned}
$$

Proof. We shall treat only the case $k \geqq 1$. The case $k=0$ will be analogously proved. From the exact sequence of cohomology groups in the stable range of dimensions: 
$\longrightarrow H^{+}\left(B_{k}, Z\right) \stackrel{\rho^{*}}{\longrightarrow} H^{+}\left(\tilde{K}_{k}, Z\right) \stackrel{i^{*}}{\longrightarrow} H^{+}\left(F_{k}, Z\right) \stackrel{{ }^{\top}}{\longrightarrow} H^{+}\left(B_{k}, Z\right) \longrightarrow$

it follows that there exists such a class $v_{k}$ as satisfying the first condition of Lemma 6. $2_{k}$. Then $\left(v_{k}\right)_{p} \in \operatorname{Im} \rho^{*}$. But $\rho^{*} H^{N_{k}+2 p^{k}(p-1)}\left(B_{k}, Z_{p}\right)$ is generated by a single element $\mathfrak{P}^{p^{k}}\left(\iota_{k}\right) p$. Therefore we can set

$$
\left(v_{k}\right)_{p}=\nu \mathfrak{P}^{p^{k}}\left(\bar{\iota}_{k}\right)_{p}, \quad \nu \in Z_{p} .
$$

From the condition (1) of the lemma, it follows that

$$
\Delta_{i}\left(v_{k}\right)_{p}=\left\{\left(\eta_{k}\right)_{p}\right\} \quad \bmod \operatorname{Im} i^{*}+\operatorname{Im} \Delta .
$$

On the other hand we know

$$
\Delta_{i}\left(\mathfrak{P}^{p^{k}}\left(\bar{\iota}_{k}\right)_{p}\right)=\left\{\left(\eta_{k}\right)_{p}\right\} \bmod \operatorname{Im} i^{*}+\operatorname{Im} \Delta .
$$

It follows that $\nu=1$ in (6.3), and this proves the condition (2).

For the next lemmas, let $y_{j} \in H^{2}\left(P^{p}, Z\right)(j=1, \cdots \cdots, p)$ be those generators of the cohomology ring $H^{*}\left(P^{p}, Z\right)$ which correspond to a fixed generator $y \in H^{*}(P, Z)$ by the diagonal map $d: P \rightarrow P^{p}$.

Lemma 6. $3_{0}$. There exists a map $g_{0}: P^{p} \rightarrow \widetilde{K}_{0}$ such that

$$
\begin{aligned}
g_{0} * \bar{\iota}_{0}= & y_{1}{ }^{n} \ldots \cdots y_{p}{ }^{n} \\
g_{0} * v_{0},{ }_{1}= & n \sum y_{1}{ }^{n+p-1} y_{2}{ }^{n} \ldots \ldots y_{p}{ }^{n} \\
g_{0}{ }^{*} v_{0},{ }_{2}= & n(n-1) / 2 \cdot \sum y_{1}{ }^{n+2(p-1)} y_{2}{ }^{n} \cdots \cdots y_{p}{ }^{n} \\
& +n^{2} \sum y_{1}{ }^{n+p-1} y_{2}{ }^{n+p-1} y_{3}{ }^{n} \cdots \cdots y_{p}{ }^{n} .
\end{aligned}
$$

Lemma 6. $3_{k}$. For each $k \geqq 1$, there exists a map $g_{k}: P^{p} \rightarrow \widetilde{K}_{k}$ such that

$$
\begin{aligned}
& g_{k} *_{\iota_{k}}=y_{1}{ }^{p k_{n}} \ldots \ldots y_{p}{ }^{p k_{n}}, \\
& g_{k}{ }^{*} v_{k}=n \sum y_{1}{ }^{{ }^{k} k_{(n+p-1)}} y_{2}{ }^{{ }^{k} k_{n}} \ldots \ldots y_{p}{ }^{p^{k_{n}}} \text {. }
\end{aligned}
$$

In these lemmas the right-hand sides of the respective formulas are the symmetric polynomials of $y_{1}, \cdots \cdots, y_{p}$ having the indicated monomial as a representative, and all cohomology classes involved are of integral coefficients.

Proof. Since Lemmas $6.3_{0}$ and $6.3_{k}$ are similarly proved, we treat only the case $k \geqq 1$. As is easily seen, there exists a map $g^{\prime}: P^{p} \rightarrow \widetilde{K}_{k}$ such that the condition (1) is fulfilled. From Lemma $6.2_{k}$, (2) it follows that

$$
g^{\prime *} v_{k}=n \sum y_{1}{ }^{p^{k}(n+p-1)} y_{2}{ }^{p^{k}} \ldots \ldots \cdot y_{p}{ }^{p^{k}}+p w,
$$

where $w$ is a suitable element of $H^{N_{k+2} p^{k}(p-1)}\left(P^{p}, Z\right)$.

Let $h: P^{p} \rightarrow K\left(Z, N_{k}+2 p^{k}(p-1)\right) \subset F_{k}$ be a map such that

$$
h^{*} \eta_{k}=-w \text {. }
$$

Set $g_{k}=h \cdot g^{\prime}$, where - means the multiplication of the principal fibre space $\left(F_{k}, \tilde{K}_{k}, B_{k}\right)$. Then the map $g_{k}$ satisfies the condition (2).

Now we will return to the proof of Theorem $4.3_{k}$. Set $f_{k}=g_{k} \circ d$, the composition of the diagonal map $d: P \rightarrow P^{p}$ and of the map $g_{k}: P^{p} \rightarrow \widetilde{K}_{k}$ appeared in Lemma $6.3_{k}$. Then we have and

$$
f_{k} * \bar{\iota}_{k}=y^{p k+1} n \quad(k \geqq 0),
$$

$$
\begin{aligned}
& f_{0}^{*} v_{0,1}=p n y^{p_{n+(p-1)}} \\
& f_{0}^{*} v_{0,2}=p n(p n-1) / 2 y^{p_{n+2(p-1)}}
\end{aligned}
$$




$$
f_{k}^{*} v_{k}=p n y^{p^{k+1} n+k^{k}(p-1)} \quad(k \geqq 1)
$$

By Lemma 6.1, we have

$$
\begin{aligned}
& \Delta_{f_{0}}\left(v_{0,1}\right)_{p}=\left\{\left(n y^{p_{n+(p-1)}}\right)_{p}\right\} \bmod \operatorname{Im} f_{0}^{*}, \\
& \Delta_{f_{0}}\left(v_{0,2}\right)_{p}=\left\{\left(n(p n-1) / 2 y^{p_{n+2(p-1)}}\right)_{p}\right\} \bmod \operatorname{Im} f_{0}^{*} \\
& \Delta_{f_{k}}\left(v_{k}\right)_{\eta}=\left\{\left(n y^{\left.\left.p k+1_{n+1} k_{(p-1)}\right)_{p}\right\}} \bmod \operatorname{Im} f_{k}{ }^{*} .\right.\right.
\end{aligned}
$$

Recalling that the operation $\Phi_{0}^{(1)}$ corresponds to the relation

$$
\left(\triangle \mathfrak{P}^{1}-2 \mathfrak{P}^{1} \Delta\right) \mathfrak{P}^{1}-2 \mathfrak{P}^{2} \Delta=0
$$

and that $\Phi_{-1 k}$ corresponds to the relation

$$
c(\Delta) c\left(\mathfrak{P}^{k^{k}}\right)-c\left(\Delta \mathfrak{P}^{x^{k}}\right) c\left(\mathfrak{P}^{1}\right)-c\left(\mathfrak{F}^{z^{k}}\right) c(\Delta)=0,
$$

and applying Theorem 1.4, we have

$$
\begin{aligned}
\Phi_{0}^{(1)}\left(y^{p_{n}}\right)_{p} & =\Phi_{0}^{(1)} f_{0} *\left(\bar{\iota}_{0}\right)_{p}=-\left(\Delta \mathfrak{P}^{1}-2 \mathfrak{P}^{1} \Delta\right)_{f_{0}} \mathfrak{P}^{\prime}\left(\bar{\iota}_{0}\right) p \\
& =2 \mathfrak{F}^{1} \Delta_{f_{0}}\left(\mathfrak{P}^{1}\left(\bar{\iota}_{0}\right) p\right)-2 \Delta_{f} \mathfrak{P}^{2}\left(\bar{\iota}_{0}\right)_{p} \\
& =2 \mathfrak{P}^{1} \Delta_{f 0}\left(v_{0},{ }_{1}\right) p-2 \Delta_{f_{0}}\left(v_{0},{ }_{2}\right) p \\
& =\left(-n y^{\left.p_{n+2(p-1)}\right) p} \bmod \operatorname{Im} f_{0}^{*}\right.
\end{aligned}
$$

and

$$
\begin{gathered}
\Phi_{-1, k}\left(y^{p k+1_{n}}\right)_{p}=\Phi_{-1}, f_{k} f_{k}^{*}\left(\bar{\iota}_{k}\right) p=-c(\Delta)_{f_{k}}\left(c\left(\mathfrak{P}^{x^{k}}\right)\left(\bar{\iota}_{k}\right)_{p}\right) \\
=-\Delta_{f_{k}}\left(\mathfrak{P}^{p k}\left(\bar{\iota}_{k}\right)_{p}\right)=-\Delta_{f_{k}}\left(v_{k}\right)_{p} \\
=\left(-n y^{\left.p k+1_{n+p} k_{(p-1)}\right) p} \bmod \operatorname{Im} f_{k}{ }^{*} .\right.
\end{gathered}
$$

It is easy to see $\operatorname{Im} f_{0} * \cap H^{N_{0}+4(p-1)}\left(P, Z_{p}\right)=0$. We shall prove

$$
\operatorname{Im} f_{k}^{*} \cap H^{N_{k}+2 \gamma^{k}(p-1)}\left(P, Z_{p}\right)=0
$$

by induction on $k$.

Lemma 6.4. In the range of degrees less than $2 N_{k}(k \geqq 1)$ the graded $A$ module $H^{+}\left(\tilde{K}_{k}, Z_{p}\right)$ is generated by the classes $\left(\left(\bar{\iota}_{k}\right)_{p}\right)$ and $\Phi_{Z *}\left(\left(\bar{\iota}_{k}\right)_{p}\right)\left(Z_{*}\right.$ run over all the basic d-cycles belonging to $C_{k-1}$ ).

This lemma is easily proved. Now assume that Theorem $4.3_{k}$ is true for smaller values of $k$. Then, from Lemma 6.4, we can prove

$$
\operatorname{Im} f_{k}^{*} \cap H^{N_{k}+2 t_{(j)-1)}}\left(P, Z_{p}\right)=0 \text {, }
$$

and this completes the proof of Theorem $4.3_{k}$.

\section{\$7. Second proof.}

We shall first establish a formula on $\Phi_{-1},{ }_{k} c\left(\mathfrak{P}^{x^{k}(p-1)}\right)$.

Let us take as left $A$-free modules $C_{0}, C_{0}{ }^{\prime}, C_{1}$ and $C_{1}{ }^{\prime}$ in $\S 1$ the following modules respectively: $A, A, C_{1}{ }^{k}(k \geqq 1), C_{1}{ }^{p-1} \oplus A\left[c \mathfrak{P}^{z^{k}} \mathfrak{P}^{p^{k-1}}\right]$. Furthermore, define $A$-map $d^{\prime}$ of $C_{1}{ }^{k-1} \oplus A\left[c \mathfrak{P}^{p^{k}} \mathfrak{P}^{p^{k-1}}\right]$ into $A$ as follows :

$$
\begin{aligned}
& d^{\prime}\left[c \mathfrak{P}^{p^{i}}\right]=c\left(\mathfrak{F}^{p^{i}}\right) \quad i=0,1, \cdots \cdots, k-1, \\
& d^{\prime}[c \Delta]=c(\Delta) \\
& d^{\prime}\left[c \mathfrak{P}^{p^{k}} \mathfrak{P}^{p^{k-1}}\right]=c\left(\mathfrak{P}^{p k} \mathfrak{P}^{p k-1}\right) .
\end{aligned}
$$

Now, let $m_{0}$ be the $A$-map of $C_{0}=A$ into $C_{0}{ }^{\prime}=A$ which is defined by $m_{0}(1)=c\left(\mathfrak{P}^{p^{k}(p-1)}\right)$. Then by a suitable choice of $A$-map $m_{1}$ of $C_{1}{ }^{k}$ into $C_{1}^{k-1} \oplus A\left[c \Re^{p^{k}} \Re^{k-1}\right]$, we can obtain the following commutative diagram 


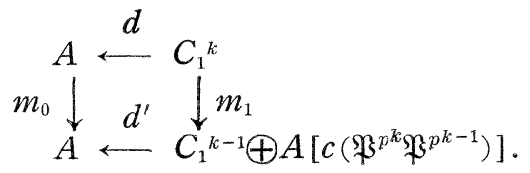

More precisely, we shall define $m_{1}$ as follows for later use. Let us begin by computing the effect of $m_{0} d$ for $\left[c \mathfrak{P}^{p^{k}}\right]$ :

$$
\begin{aligned}
m_{0} d\left[c \mathfrak{P}^{p^{k}}\right] & =m_{0} c\left(\mathfrak{P}^{p^{k}}\right) \\
& =c\left(\mathfrak{P}^{p^{k}}\right) c\left(\mathfrak{P}^{p^{k}(p-1)}\right) \\
& =c\left(\mathfrak{P}\left(p^{k}(p-1), p^{k}\right)\right) .
\end{aligned}
$$

Now, by the Adem relation we have

$$
\begin{aligned}
& \mathfrak{P}\left(p^{k}(p-1), p^{k}\right) \equiv \sum_{i=1}^{p-1}(-1)^{i} \mathfrak{P}\left(p^{k}(p-1)+(p-i) p^{k-1}, i p^{k-1}\right) \quad \bmod M_{*}{ }^{\prime}(k-2) \\
& \equiv \sum_{i=1}^{p-1} \frac{(-1)^{i}}{p-i} \mathfrak{P}\left(p^{k-1}, p^{k}(p-1)+p^{k-1}(p-i-1), i p^{k-1}\right) \\
& \bmod M_{*}{ }^{\prime}(k-2) \\
& =\sum_{i=1}^{p-1} \frac{(-1)^{i+1}}{i} \mathfrak{B}\left(p^{k-1}, p^{k}(p-1)+p^{k-1}(p-i-1), i p^{k-1}\right) .
\end{aligned}
$$

Define $m_{1}$ for $\left[c \mathfrak{P}^{p^{k}}\right]$ as follows:

$$
\begin{aligned}
m_{1}\left[c \mathfrak{P}^{p^{k}}\right]= & \sum_{i=1}^{p-1} \frac{(-1)^{i+1}}{i} c \mathfrak{P}\left(p^{k}(p-1)+p^{k-1}(p-i-1), i p^{k-1}\right)\left[c \mathfrak{B}^{p k-1}\right] \\
& +\tau_{k}, k
\end{aligned}
$$

where $\tau_{k},{ }_{k}$ is an element of $C_{1}{ }^{k-2}$, so that $m_{0} d\left[c \mathfrak{B}^{p^{k}}\right]=d^{\prime} m_{1}\left[c \mathfrak{P}^{p^{k}}\right]$,

Similarly, define $m_{1}$ for $\left[c \mathfrak{P}^{p k-1}\right],\left[c \mathfrak{B}^{p k-2}\right]$ as follows:

$$
\begin{aligned}
& m_{1}\left[c \mathfrak{P}^{p^{k-1}}\right]=c \mathfrak{F}^{p^{k}(p-1)}\left[c\left(\mathfrak{P}^{p^{k}} \mathfrak{P}^{p^{k-1}}\right)\right]+2 c \mathfrak{P}^{k^{k_{(p-1)}}}\left[\mathfrak{P} c^{p k-1}\right]+\tau_{k-1}, k \\
& m_{1}\left[c \mathfrak{P}^{p^{k-2}}\right]=c \mathfrak{P}^{p^{k}(p-2)+p k-1(p-1)+p^{k-2}}\left[c \mathfrak{P}^{p^{k-1}}\right]+\tau_{k-2}, k
\end{aligned}
$$

where $\tau_{k-1}, k, \tau_{k-2},{ }_{k}$ may be regarded as elements of $C_{1}{ }^{k-2}$, so that $m_{0} d=d^{\prime} m_{1}$. Furthermore, for $0 \leqq j<k-2$ we can take an $m_{1}\left[c \mathfrak{F}^{p^{j}}\right]$ as element of $C_{1}{ }^{k-2}$ by Lemma 2.1 such that $m_{0} d\left[c \mathfrak{P}^{p j}\right]=d^{\prime} m_{1}\left[c \mathfrak{P}^{p^{j}}\right]$. With respect to $m_{1}[c \Delta]$, we may choose $m_{1}[c \Delta]$ in a similar way as above for $k \geqq 2$. If $k=1$, then we may define

$$
m_{1}[c \Delta] \equiv c\left(\Delta \mathfrak{P}^{p(p-2)+p-1}\right) \quad \bmod C_{1}^{-1}
$$

such that $m_{0} d[c \Delta]=d^{\prime} m_{1}[c \Delta]$.

Let us compute $m_{1} Z_{-1, k}=m_{1}\left\{c\left(\Delta\left[c \mathfrak{F}^{p^{k}}\right]-c\left(\Delta \mathfrak{P}^{p^{k}-1}\right)\left[c \mathfrak{P}^{1}\right]-c\left(\mathfrak{P}^{p^{k}}\right)[c \Delta]\right\}\right.$.

We shall now consider three cases separately.

Case I, $k \geqq 3$. We have immediately

$$
\begin{array}{rl}
m_{1} Z_{-1}, k & c c(\Delta) \sum_{i=1}^{p-1} \frac{(-1)^{i+1}}{i} c \mathfrak{P}\left(p^{k-1}(p-i-1)+p^{k}(p-1), i p^{k-1}\right)\left[c \mathfrak{P}^{p^{k-1}}\right] \\
& \bmod C_{1}{ }^{k-2} \\
= & \sum_{i=1}^{p-1} \frac{(-1)^{i+1}}{i} c \mathfrak{P}\left(p^{k-1}(p-i-1)+p^{k}(p-1), i p^{k-1}, \Delta\right)\left[c \mathfrak{P}^{p k-1}\right] .
\end{array}
$$

By the repeated applications of (1.3), we can obtain the following

(7.6) $\quad \mathfrak{P}\left(p^{k}(p-1)+p^{k-1}(p-i-1), i p^{k-1}, \Delta\right)$ 


$$
\begin{aligned}
=\mathfrak{P}\left(p^{k}(p-1)\right. & \left.+p^{k-1}(p-i-1), 1, \Delta, i p^{k-1}-1\right) \\
& +\mathfrak{P}\left(p^{k}(p-1)+p^{k-1}(p-i-1), \Delta, i p^{k-1}\right) \\
=\mathfrak{P}\left(p^{k}(p-1)\right. & \left.+p^{k-1}(p-i-1), 1, \Delta, i p^{k-1}-1\right) \\
& +\mathfrak{P}\left(1, \Delta, p^{k}(p-1)+p^{k-1}(p-i-1)-1, i p^{k-1}\right) \\
& +\mathfrak{P}\left(\Delta, p^{k}(p-1)+p^{k-1}(p-i-1), i p^{k-1}\right)
\end{aligned}
$$

$\equiv \mathfrak{P}\left(\Delta, p^{k}(p-1)+p^{k-1}(p-i-1), i p^{k-1}\right) \bmod \mathfrak{\beta}^{2 p k-2} A+M_{*}^{\prime}(1)$, because for $k>3 \mathfrak{P}\left(p^{k}(p-1)+p^{k-1}(p-i-1), 1\right)$ is contained in $\mathfrak{B}^{2} p^{k-2} A+M_{k^{\prime}}{ }^{\prime}(1)$ and, for $k=3$, it is contained in $\mathfrak{P}^{2} p A+M_{*}^{\prime}(0)$ by the Adem relation

$$
\begin{aligned}
& \mathfrak{P}\left(2 p, p^{3}(p-1)+p^{2}(p-i-1)+1-2 p\right) \\
\equiv & -\mathfrak{S}\left(p^{3}(p-1)+p^{2}(p-i-1), 1\right) \bmod M_{*}^{\prime}(0) .
\end{aligned}
$$

Thus (7.5), (7.6) imply

Proposition 7.1. We have

for $k>3 \Phi_{-1},{ }_{k} c\left(\mathfrak{P}^{p k(p-1)}\right) \equiv \sum_{i=1}^{p-1} \frac{(-1)^{i+1}}{i} c \mathfrak{P}\left(p^{k}(p-1)+p^{k-1}(p+i-1), i p^{k-1}\right) \Phi_{-1},{ }_{k-1}$

$$
\begin{aligned}
& +\lambda_{k} \mathfrak{S}^{p^{k+1}} \\
& \bmod A \Phi_{1, k-1}+A \Phi_{0, k-1}+\sum_{s<k-1} A \Phi_{r, s}+\sum_{s<k-1} A \Phi_{s}^{(r)}
\end{aligned}
$$

for $k=3 \Phi_{-1, k} c\left(\mathfrak{P}^{p^{k}(p-1)}\right) \equiv \sum_{i=1}^{p-1} \frac{(-1)^{i+1}}{i} c \mathfrak{B}\left(p^{k}(p-1)+p^{k-1}(p-i-1), i p^{k-1}\right) \Phi_{-1},{ }_{k-1}$

$$
+\lambda_{k} \mathfrak{F}^{p k+1}
$$

$$
\bmod A \Phi_{k-1}^{(0)}+A \Phi_{0, k-1}+\sum_{s<k-1} A \Phi_{r, s}+\sum_{s<k-1} A \Phi_{s}^{(r)} .
$$

Case II, $k=2$. From (7.1) and (7.3) we have

$$
\begin{aligned}
& m_{1} Z_{-1},{ }_{2} \equiv\left\{\sum_{i=1}^{p-1} \frac{(-1)^{i+1}}{i} c \mathfrak{P}\left(p^{2}(p-1)+p(p-i-1), i p, \Delta\right)\right. \\
&\left.-c \mathfrak{P}\left(p^{2}(p-2)+p(p-1)+1, \Delta, p^{2}-1\right)\right\}\left[c \mathfrak{P}^{p}\right] \bmod C_{1}{ }^{0}
\end{aligned}
$$

Now, by the repeated applications of (1.3) we can obtain the following relations

$$
\begin{aligned}
\mathfrak{P}( & \left.p^{2}(p-1)+p(p-i-1), i p, \Delta\right) \\
= & \mathfrak{P}\left(p^{2}(p-1)+p(p-i-1), 1, \Delta, i p-1\right) \\
\quad & \quad \mathfrak{P}\left(p^{2}(p-1)+p(p-i-1), \Delta, i p\right) \\
= & \mathfrak{P}\left(p^{2}(p-1)+p(p-i-1), 1, \Delta, i p-1\right)-\mathfrak{P}\left(2, \Delta, p^{2}(p-1)\right. \\
\quad & \quad+p(p-i-1)-2, i p)+\mathfrak{P}\left(\Delta, p^{2}(p-1)+p(p-i-1), i p\right) \\
\equiv & \mathfrak{P}\left(p^{2}(p-1)+p(p-i-1), 1, \Delta, i p-1\right) \\
\quad & \quad \mathfrak{P}\left(\Delta, p^{2}(p-1)+p(p-i-1), i p\right) \bmod \mathfrak{P}^{2} A .
\end{aligned}
$$

Also, by the Adem relation we have

$$
\begin{aligned}
\mathfrak{P}( & \left.p^{2}(p-2)+p(p-1)+1, \Delta, p^{2}-1\right) \\
\quad & \sum_{i=1}^{p-2}(-1)^{i+1} \mathfrak{P}\left(p^{2}(p-1)+p(p-i-2)+1, \Delta, i p+p-1\right) \\
& +\sum_{0<<p, i}(-1)^{j}(j+1)\left(\begin{array}{c}
j \\
i
\end{array}\right) \mathfrak{P}\left(\Delta, p^{2}(p-1)+p(p-i-2)+p-j, i p+j\right)
\end{aligned}
$$

$\bmod \mathfrak{B}^{2} A$. 
Thus we can obtain the following equalities.

$$
\begin{aligned}
& \sum_{i=1}^{p-1} \frac{(-1)^{i}}{i} \mathfrak{P}\left(p^{2}(p-1)+p(p-i-1), 1, \Delta, i p-1\right) \\
+ & \sum_{i=0}^{p-2}(-1)^{i+1} \mathfrak{P}\left(p^{2}(p-1)+p(p-i-2)+1, \Delta, i p+p-1\right) \\
= & \sum_{i=1}^{p-1} \frac{(-1)^{i}}{i}\left\{\mathfrak{P}\left(p^{2}(p-1)+p(p-i-1), 1, \Delta, i p-1\right)\right. \\
& +i \mathfrak{P}\left(p^{2}(p-1)+p(p-i-1)+1, \Delta, i p-1\right\} \\
\equiv & 0 \quad \bmod \left(2 \mathfrak{P}^{p+1}-\mathfrak{P}^{p} \mathfrak{P}^{1}\right) A,
\end{aligned}
$$

because $\left(2 \mathfrak{P}^{p+1}-\mathfrak{P}^{p} \mathfrak{P}^{1}\right) \mathfrak{B}\left(p^{2}(p-1)+p(p-i-2)\right)$

$$
=-\mathfrak{P}\left(p^{2}(p-1)+p(p-i-1), 1\right)-i \mathfrak{P}\left(p^{2}(p-1)+p(p-i-1)+1\right) \text {. }
$$

That is, from (7.8), (7.9) and (7.10) we have

$$
\begin{gathered}
\sum_{i=1}^{p-1} \frac{(-1)^{i+1}}{i} \mathfrak{P}\left(p^{2}(p-1)+p(p-i-1), i p, \Delta\right) \\
-\mathfrak{P}\left(p^{2}(p-2)+p(p-1)+1, \Delta, p^{2}-1\right) \\
\equiv \sum_{i=1}^{p-1} \frac{(-1)^{i+1}}{i} \mathfrak{P}\left(\Delta, p^{2}(p-1)+p(p-i-1), i p\right) \\
+\sum_{i, 0<j<p}(-1)^{j+1}(j+1)\left(\begin{array}{c}
j \\
i
\end{array}\right) \mathfrak{P}\left(\Delta, p^{2}(p-1)+p(p-i-2)+p-j, i p+j\right) \\
\bmod \mathfrak{P}^{2} A+\left(2 \mathfrak{P}^{p+1}-\mathfrak{P}^{p} \mathfrak{P}^{1}\right) A .
\end{gathered}
$$

(7.7) and (7.11) imply

Proposition 7.2. $\Phi_{-1},{ }_{2} c\left(\mathfrak{P}^{p^{2}(p-1)}\right)$

$$
\begin{aligned}
& \quad \equiv\left\{\sum_{i=1}^{p-1} \frac{(-1)^{i+1}}{i} c \mathfrak{P}\left(p^{2}(p-1)+p(p-i-1), i p\right)\right. \\
& \left.+\sum_{i, 0<j<p}(-1)^{j+1}(j+1)\left(\begin{array}{c}
j \\
i
\end{array}\right) c \mathfrak{B}\left(p^{2}(p-1)+p(p-i-2)+p-j, i p+j\right)\right\} \Phi_{-1,1} \\
& +\lambda_{2} \mathfrak{P}^{p^{3}} \bmod A \Phi_{1}^{(0)}+A \Phi_{1}^{(1)}+A \Phi_{0}^{(1)}+A \Phi_{0}^{(2)}+A \Phi_{-1,-1} .
\end{aligned}
$$

Case III, $k=1$. Let us begin with specifying basic $d^{\prime}$-cycles of $C_{1}{ }^{0} \oplus A\left[c\left(\mathfrak{P}^{p} \mathfrak{P}^{1}\right)\right]$. Define $\lambda, \mu$ by

$$
\begin{aligned}
& \lambda=c(R)\left[c\left(\mathfrak{P}^{p} \mathfrak{P}^{1}\right)\right]+2 c\left(\mathfrak{P}^{p+2}-\mathfrak{P}^{p+1} \mathfrak{P}^{1}\right)[c \Delta] \\
& \mu=c\left(\mathfrak{P}^{1}\right)\left[c\left(\mathfrak{P}^{p} \mathfrak{P}^{1}\right)\right]+c\left(-2 \mathfrak{P}^{p} \mathfrak{P}^{1}+\mathfrak{P}^{p+1}\right)\left[c \mathfrak{P}^{1}\right]
\end{aligned}
$$

where $R=2 \Delta \mathfrak{P}^{1}-\mathfrak{P}^{1} \Delta$. Furthermore, denote the stable secondary cohomology operations associated with $\left(d^{\prime}, \lambda\right)$ and $\left(d^{\prime}, \mu\right)$ by $\Phi_{\lambda}$ and $\Phi_{\mu}$ respectively. Then $\Phi_{\lambda}$ and $\Phi_{\mu}$ which are defined for $\operatorname{Ker} \mathfrak{F}^{p+1} \cap \operatorname{Ker} \mathfrak{P}^{1} \cap \operatorname{Ker} \Delta$ are uniquely determined operations, because $A / d C_{1}{ }^{\prime}=A / d^{\prime}\left(C_{1} \oplus A\left[c\left(\mathfrak{P}^{p} \mathfrak{P}^{1}\right)\right]\right)$ is zero in degrees $2(p+2)(p-1), 2(p+2)(p-1)-1$ (Cf. Theorem 1.1(2)).

Now, by $(7.1),(7.2)$ and $(7.4)$ we have

$$
\begin{aligned}
m_{1} Z_{-1}, 1 \equiv & -c \mathfrak{P}(p(p-2), \Delta, p-1)\left[c\left(\mathfrak{P}^{p} \mathfrak{P}^{1}\right)\right] \\
& +\left\{\sum_{i=1}^{p-1} \frac{(-1)^{i+1}}{i} c \mathfrak{P}(p(p-1)+p-i-1, i, \Delta)\right. \\
& -2 c \mathfrak{P}(p(p-1), \Delta, p-1)
\end{aligned}
$$




$$
-c \mathfrak{P}(\Delta, p(p-2)+p-1, p)\}\left[c \mathfrak{B}^{1}\right] \bmod C_{1}^{-1}
$$

Next, we shall express $m_{1} Z_{-1,1}$ as an element of $A \lambda+A \mu+A W_{0}+A U_{0} \bmod C_{1}^{-1}$. By the Adem relation we obtain

$$
\mathfrak{P}(p(p-2), \Delta, p-1)=\sum_{i=0}^{p-2}(-1)^{i+1} \mathfrak{P}(\Delta, p(p-2)+p-i-1, i) .
$$

Further, utilizing $1 / 2\left(R+\mathfrak{P}^{1} \Delta\right) \mathfrak{P}^{s}=(s+1) \mathfrak{P}(\Delta, s+1)$, we have

$$
\mathfrak{P}(p(p-2), \Delta, p-1)=\frac{1}{2} \sum_{i=0}^{p-2} \frac{(-1)^{i}}{i+1}\left(R+\mathfrak{P}^{1} \Delta\right) \mathfrak{P}(p(p-2)+p-i-2, i) .
$$

Thus, our object will be attained by expressing the element

$$
\begin{aligned}
z= & \frac{1}{2} \sum_{i=0}^{p-2} \frac{(-1)^{i}}{i+1}\left(\mathfrak{P}^{p+1}-2 \mathfrak{P}^{p} \mathfrak{P}^{1}\right) \mathfrak{P}(\Delta, p(p-2)+p-i-2, i) \\
& \left.+\sum_{i=1}^{p-2} \frac{(-1)^{i+1}}{i} \mathfrak{P}(p(p-1)+p-i-1, i, \Delta)-2 \mathfrak{P}(p-1), \Delta, p-1\right) \\
& -\mathfrak{P}(\Delta, p(p-2)+p-1, p)
\end{aligned}
$$

as an element of $R A+\mathfrak{P}^{p-1} A$. By the Adem relation we have

$$
\begin{aligned}
z= & \frac{1}{2} \sum_{i=0}^{p-2} \frac{(-1)^{i}}{i+1}\{(i-1)(i+2) \mathfrak{P}(\Delta, p(p-1)+p-i-1, i) \\
& +(i+1)^{2} \mathfrak{P}(\Delta, p(p-1)+p-i-2, i+1) \\
& -(i-1) \mathfrak{P}(p(p-1)+p-i-2, \Delta, i+1) \\
& -(i-1) \mathfrak{P}(p(p-1)+p-i-1, \Delta, i)\} \\
& -2 \mathfrak{P}(p(p-1), \Delta, p-1)+\mathfrak{B}(\Delta, p(p-1)+p-1) \\
= & \sum_{i=1}^{p-2} \frac{(-1)^{i}}{i(i+1)} R \mathfrak{P}(p(p-1)+p-i-2, i)-\frac{1}{2} R \mathfrak{P}(p(p-1)+p-2) .
\end{aligned}
$$

Thus we obtain finally

$$
\begin{aligned}
m_{1} Z_{-1,1} \equiv & \frac{1}{2} \sum_{i=0}^{p-1} \frac{(-1)^{i+1}}{i+1} c \mathfrak{P}(p(p-2)+p-i-2, i)(\lambda+c(\Delta) \mu) \\
& +\left[\sum_{i=1}^{p-2} \frac{(-1)^{i}}{i(i+1)} c \mathfrak{P}(p(p-1)+p-i-2, i)-\frac{1}{2} c \mathfrak{P}\left(p(p-1)+p-2 \mid V_{0}\right.\right. \\
& \bmod C_{1}^{-1} .
\end{aligned}
$$

This implies the following proposition.

Probosition 7.3. $\Phi_{-1},{ }_{1} c \Re^{p(p-1)}$

$$
\begin{aligned}
& =\frac{1}{2} \sum_{i=0}^{p-2} \frac{(-1)^{i+1}}{i+1} c \mathfrak{P}(p(p-2)+p-i-2, i)\left(\Phi_{\lambda}+c(\Delta) \Phi_{\mu}\right) \\
& +\left[\sum_{i=1}^{p-2} \frac{(-1)^{i}}{i(i+1)} c \mathfrak{P}(p(p-1)+p-i-2, i)-\frac{1}{2} c \mathfrak{P}(p(p-1)+p-2] \Phi_{0}^{(1)}\right. \\
& +\lambda_{1} \mathfrak{P}^{p^{2}} \bmod A \Phi_{-1,-1}
\end{aligned}
$$

REMARK 7.4. In the same manner as in $\$ 3$ we shall define, in case $p=2$, cycles in $C_{1}^{k}=\sum_{i=0}^{k} A\left[\mathrm{Sq}^{2 i}\right]$, which are contained in the kernel of $A$-map $d$

$$
d\left[c \mathrm{Sq}^{2^{i}}\right]=c\left(\mathrm{Sq}^{2^{i}}\right)(i=0, \cdots \cdots, k)
$$

as follows:

$$
z_{i, j}=c\left(\mathrm{Sq}^{2^{i}}\right)\left[c \mathrm{Sq}^{2^{j}}\right]+\cdots \cdots, i \neq j-1, i \leqq j
$$


Then we have a similar result to Proposition 3.2 which is stated as follows: The kernel of $d: C_{1}{ }^{k} \rightarrow A$ is just $\sum_{\substack{i \leq j \leq k \\ i \neq j=1}} A z_{i, j}$ (Cf. [10]). Also if we define

$$
z_{0, k}=c\left(\mathrm{Sq}^{1}\right)\left[c \mathrm{Sq}^{2^{k}}\right]+c\left[\mathrm{Sq}^{2^{k}-1}\right)\left[c \mathrm{Sq}^{2}\right]+c\left(\mathrm{Sq}^{2^{k}}\right)\left[c \mathrm{Sq}^{1}\right] \quad(k \geqq 2),
$$

then we can obtain

$$
\begin{aligned}
& \text { for } k>3 \quad \Phi_{0, k} c\left(\mathrm{Sq}^{2}\right)=c\left(\mathrm{Sq}^{2 k} \mathrm{Sq}^{2^{k-1}}\right) \Phi_{0, k-1}+\sum_{i \neq 0} \alpha_{i},{ }_{k-1} \Phi_{k-1} \\
& +\sum_{j \leqq k-2} \alpha_{i}, \Phi_{i, j}+\lambda_{k}^{\prime} \mathrm{Sq}^{2^{k+1}} \\
& \text { for } k=3 \quad \Phi_{0, k} c\left(\mathrm{Sq}^{2 k}\right)=c\left(\mathrm{Sq}^{2^{k}} \mathrm{Sq}^{2^{k-1}}+\mathrm{Sq}^{2^{k+2} 2_{k-1}}+\mathrm{Sq}^{2^{k}+2^{k-2}} \mathrm{Sq}^{2^{k-2}}\right) \Phi_{0, k-1} \\
& +\sum_{i \neq 0} \alpha_{i, k-1}^{\prime} \Phi_{i k-1}+\sum_{j \leqq k-1} \alpha_{i, j}^{\prime} \Phi_{i, j}+\lambda_{3}^{\prime} \mathrm{Sq}^{2^{k+1}} .
\end{aligned}
$$

Now, Let us compute $\Phi_{0}{ }^{(1)} y^{p_{n}}$. For our purpose we refer to Toda's result [17] which may be stated in basic operations as follows.

(7.14)

$\mathfrak{B}^{p}=-\Delta \Phi_{0}^{(2)}-\mathfrak{P}^{p-2} \Phi_{0}{ }^{(1)}$.

Apply this theorem for $y^{p_{n}}$, then we obtain

$$
\mathfrak{P}^{p} y^{p_{n}}=n y^{p(n+p-1)}=-\mathfrak{P}^{p-2} \Phi_{0}^{(1)} y^{p_{n}} .
$$

This implies Theorem $4.3_{0}$ :

$$
\Phi_{0}^{(1)} y^{p_{n}}=-n y^{p_{n+2(p-1)}} \quad \bmod z e r o .
$$

Next, we shall consider effects of $\Phi_{\lambda}$ and $\Phi_{\mu}$ which are defined for a cohomology class $y^{p_{n}}$. It is easily verified that $y^{p_{n}}$ is contained in $\operatorname{Ker} \mathfrak{P}^{p+1}$ $\cap \operatorname{Ker} \mathfrak{P}^{1} \cap \operatorname{Ker} \Delta$. Obviously $\Phi_{\mu}$ operates on $y^{p_{n}}$ trivially as $H^{2 j+1}\left(P, Z_{p}\right)=0$. Thus we are only interested in $\Phi_{\lambda} y^{p_{n}} . \Phi_{\lambda} y^{p_{n}}$ is determined with indeterminacy of the following subgroup

$$
\operatorname{Im} c(R)+\operatorname{Im} c\left(\mathfrak{P}^{p+2}-\mathfrak{P}^{p+1} \mathfrak{P}^{1}\right) .
$$

The first term is zero, as $c(R)$ 'lifts' the dimension by $2(p-1)+1$. As for the second term, we notice

$$
c\left(\mathfrak{P}^{p+2}-\mathfrak{P}^{p+1} \mathfrak{P}^{1}\right) y^{p_{n}}=\left(-\mathfrak{P}^{p} \mathfrak{P}^{2}+\mathfrak{P}^{1} \mathfrak{P}^{p} \mathfrak{P}^{1}\right) y^{p_{n}}=0
$$

Thus $\Phi_{\lambda} y^{p_{n}}$ is settled without any indeterminacy.

LEMMA 7.5. We obtain

$$
\Phi_{0}{ }^{(1)} c\left(\mathfrak{P}^{p}\right)=\Phi_{\lambda} .
$$

Proof. In Theorem 1.1(4), put $C_{0}=C_{0}{ }^{\prime}=A, C_{1}=C_{1}{ }^{0}$ and $C_{1}{ }^{\prime}=C_{1}{ }^{0}$

$\oplus A\left[c\left(\mathfrak{P}^{p} \mathfrak{P}^{1}\right]\right.$. Let $m_{0}$ be the $A$-map defined by $m_{0}(1)=c\left(\mathfrak{P}^{p}\right)$ and $m_{1}$ the $A$ map defined as follows:

$$
\begin{aligned}
& m_{1}\left[c \mathfrak{F}^{1}\right]=\left[c\left(\mathfrak{P}^{p} \mathfrak{P}^{1}\right]\right. \\
& m_{1}[c \Delta]=c\left(\Delta \mathfrak{P}^{p-1}\right)\left[c \mathfrak{P}^{1}\right]+c \mathfrak{P}^{p}[c \Delta] .
\end{aligned}
$$

Then we have the following commutative diagram

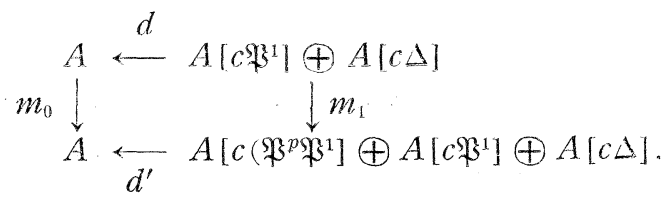

Consider $m_{1} V_{0}=m_{1}\left\{c(R)\left[c \mathfrak{B}^{1}\right]-2 c\left(\mathfrak{P}^{2}\right)[c \Delta]\right\}$

$$
=c(R)\left[c\left(\mathfrak{P}^{p} \mathfrak{B}^{1}\right)\right]-2 c\left(\mathfrak{P}^{2}\right) c\left(\Delta \mathfrak{P}^{p-1}\right)\left[c \mathfrak{P}^{1}\right]-2 c\left(\mathfrak{P}^{2}\right) c\left(\mathfrak{P}^{p}\right)[c \Delta]
$$




$$
\begin{aligned}
& =c(R)\left[c\left(\mathfrak{P}^{p} \mathfrak{P}^{1}\right)\right]+2 c\left(\mathfrak{P}^{p+2}-\mathfrak{P}^{p+1} \mathfrak{F}^{1}\right)[c \Delta] \\
& =\lambda .
\end{aligned}
$$

On the other hand, $\Phi_{\lambda}$ is a uniquely determined operation. Therefore our lemma is proved by utilizing Theorem 1.1 (4).

Now, we shall prove the following proposition by making an essential use of Lemma 7.5 and Theorem 4.3.

Proposition 7.2. $\Phi_{\lambda} y^{p_{n}}=n(n-1) y^{p_{n+(p+2)(p-1)}} \bmod z$ zero.

Proof. The computation of $\Phi_{\lambda} y^{p_{n}}$ is performed as follows:

$$
\begin{aligned}
\Phi_{\lambda} y^{p_{n}} & =\Phi_{0}^{(1)} c\left(\mathfrak{P}^{p}\right) y^{p_{n}=-} \Phi_{0}{ }^{(1)} \mathfrak{S}^{p} y^{p_{n}} \\
& =-n \Phi_{0}^{(1)} y^{p_{(n+p-1)}} \\
& =n(n-1) y^{p_{n+(p+2)(p-1)}} .
\end{aligned}
$$

Next we shall give the effect of $\Phi_{-1}, 1$ for $y^{p^{2}}$. For our purpose, we shall first compute $\Phi_{-1},{ }_{1} y^{p^{2}(p-1)}$. By $\mathfrak{P}^{p(p-1)} y^{p(p-1)}=y^{p^{2}(p-1)}$, by Lemma 2.4, and $M^{\prime}(0) y^{p(p-1)}=0$ we have

$$
\begin{aligned}
\Phi_{-1,1} y^{\mathfrak{p}^{2}(p-1)} & =\Phi_{-1,}, \mathfrak{P}^{p(p-1)} y^{p(p-1)} \\
& =\Phi_{-1},{ }_{1} c\left(\mathfrak{P}^{p(p-1)}\right) y^{p(p-1)}
\end{aligned}
$$

Making use of Proposition 7.3, we have

$$
\begin{aligned}
& \Phi_{-1,1} c\left(\mathfrak{P}^{p(p-1)}\right) y^{p(p-1)} \\
& =\frac{1}{2} \sum_{i=0}^{p-2} \frac{(-1)^{i+1}}{i+1} c \mathfrak{P}(p(p-2)+p-i-2, i) \Phi_{\lambda} y^{p(p-1)} \\
& +\sum_{i=1}^{p-2} \frac{(-1)^{i}}{i(i+1)} c \mathfrak{P}(p(p-1)+p-i-2, i)-\frac{1}{2} c \mathfrak{P}(p(p-1+p-2)) \\
& \times \Phi_{0}^{(1)} y^{p(p-1)}+\lambda_{1} \mathfrak{P}^{p^{2}} y^{p(p-1)} \text { mod zero. }
\end{aligned}
$$

By Proposition 7.6 we have $\Phi_{\lambda} y^{p(p-1)}=2 y^{2(p+1)(p-1)}$. Moreover, we have $c \mathfrak{P}(p(p-2)+p-i-2, i)=c \mathfrak{P}(p-i-2, p(p-2), i)$

$$
\begin{aligned}
& =c\left(\mathfrak{P}^{i}\right) c\left(\mathfrak{P}^{p(p-2}\right) c\left(\mathfrak{P}^{p-i-2}\right) \\
& =-\mathfrak{P}^{i} c\left(\mathfrak{P}^{p(p-2)}\right) \mathfrak{P}^{p-i-2} .
\end{aligned}
$$

Thus $\quad c \mathfrak{B}(p(p-2)+p-i-2, i) y^{2(p+1)(p-1)}$

Here we have

$$
=(-1)^{i+1}(i+1) \mathfrak{P}^{i} c\left(\mathfrak{P}^{p(p-2)}\right) y^{(3 p-i)(p-1)} \text {. }
$$

$$
\begin{aligned}
\mathfrak{P}^{i} c\left(\mathfrak{P}^{p(p-2)}\right) y^{(3 p-i(p-1)} \\
=-\mathfrak{P}^{i} \mathfrak{P}^{p(p-2} y^{\left(3^{p-i)(p-1)}\right.} \\
\quad=-\left(\begin{array}{c}
2 p-i-3 \\
p-2
\end{array}\right) \mathfrak{P}^{i} y^{\left(p^{2}+p-i\right)(p-1)}
\end{aligned}
$$

by successive applications of

$$
\begin{aligned}
-j \mathfrak{P}^{i} c\left(\mathfrak{P}^{p(p-j)}\right) y^{l p-i} & =\mathfrak{P}^{i} c\left(\mathfrak{P}^{p} \mathfrak{P}^{\left.p^{(p-j-1)}\right)} y^{p^{-i}}\right. \\
& =\mathfrak{P}^{i} c\left(\mathfrak{P}^{p(p-j-1)}\right) c\left(\mathfrak{P}^{p}\right) y^{p^{p-1}} \quad(0<j<p) .
\end{aligned}
$$

Therefore, if $i \neq p-2$, the right-hand side of (7.18) is equal to zero. Continuing the computation, we have

$$
\frac{1}{2} \sum_{i=0}^{p-1} \frac{(-1)^{i+1}}{i+1} c \mathfrak{P}(p(p-2)+p-i-2, i) \Phi_{\lambda} y^{p(p-1)}=y^{p(p-1)(p+1)} \text {. }
$$


In the same manner as above, we can obtain the result that the second term of the right-hand side in (7.17) is equal to zero, and that $\mathfrak{P}^{p^{2}} y^{p(p-1)}=0$. Finally we have

$$
\Phi_{-1},{ }_{1} y^{p^{2}(p-1)}=y^{p(p-1)(p+1)} .
$$

Then we have the first part of Theorem 5.2 (see Proof of Theorem 5.2). From this theorem we can easily deduce Theorem $4.3_{1}$ :

$$
\Phi_{-1,1} y^{p^{2} n}=-n y^{p\left(p_{n}+p-1\right)} \bmod z e r o,
$$

by operating the both sides of Theorem $5.2(k=1)$ on $y^{p^{2}}$.

Now, we shall prove Theorem $5.4_{k}$ by induction on $k$.

Assume that our theorem is proved for $j<k$, and notice that we have then for $j<k-1$

$\Phi_{-1, j} y^{p k_{n}}=0$ without any modulus. Our first object will be

$$
\Phi_{-1, k} p^{p k-1(p-1)}=y^{p k(p-1)(p-1)}
$$

Now we have $\mathfrak{P}^{p^{k}(p-1)} y^{p^{k}(p-1)}=y^{p k+1(p-1)}$, and $M^{\prime}(k-1) y^{p k(p-1)}=0$. So we have

$$
\begin{aligned}
\Phi_{-1},{ }_{k} y^{p k+1}(p-1) & =\Phi_{-1}, \mathfrak{F}^{p k_{(p-1)}} y^{p^{k}(p-1)} \\
& =\Phi_{-1},{ }_{k} c\left(\mathfrak{F}^{p^{k}(p-1)}\right) y^{p^{k}(p-1)}
\end{aligned}
$$

Hence, making use of Theorem 4.3, the hypothesis of induction and Proposition $7.1(k \geqq 3)$ or Proposition $7.2(k=2)$, we have

(7.23) for $k=2 \quad \Phi_{-1},{ }_{2} y^{p^{3}(p-1)}$

$$
\begin{aligned}
& =\left\{\sum_{i=1}^{p-1} \frac{(-1)^{i+1}}{i} c \mathfrak{P}\left(p^{2}(p-1)+p(p-i-1), i\right)\right. \\
& \left.+\underset{i, 0<j<p}{ }(-1)^{j+1}(j+1)\left(\begin{array}{c}
j \\
i
\end{array}\right) c \mathfrak{P}\left(p^{2}(p-1)+p(p-i-2)+p-j, i p+j\right)\right\} \\
& \times \Phi_{-1}, k-1 y^{p^{2}(p-1)}+\lambda_{k} \mathfrak{P}^{3} y^{p^{2}(p-1)} \quad \bmod \text { zero, }
\end{aligned}
$$

(7.24) for $k \geqq 3 \quad \Phi_{-1, k} y^{p k+1(p-1)}$

$$
\begin{aligned}
& =\sum_{i=1}^{p-1} \frac{(-1)^{i+1}}{i} c \mathfrak{P}\left(p^{k}(p-1)+p^{k-1}(p-i-1), i p^{k-1}\right) \Phi_{-1, k-1} y^{p^{k}(r-1)} \\
& +\lambda_{k} \mathfrak{P}^{p^{k+1}} y^{p k(p-1)} \bmod \text { zero. }
\end{aligned}
$$

Now, let us compute as follows.

$$
\begin{aligned}
& c \mathfrak{P}(\left.p^{2}(p-1)+p(p-i-2)+p-j, i p+j\right) \Phi_{-1, k-1} y^{p k_{(p-1)}} \\
&=c \mathfrak{P}\left(p-j, p^{2}(p-1)+p(p-i-2), i p+j\right) \Phi_{-1, k-1} y^{p k_{(p-1)}} \\
&=(-1)^{j} c \mathfrak{P}\left(p^{2}(p-1)+p(p-i-2), i p+j\right) \mathfrak{P}^{p-j} y^{p k-1}(p+1)(p-1) \\
& \quad=0 \quad(p>j>0) .
\end{aligned}
$$

Thus we have for $k \geqq 2$

$$
\begin{aligned}
\Phi_{1},{ }_{k} & y^{p k+1}(p-1) \\
& =\sum_{i=1}^{p-1} \frac{(-1)^{i+1}}{i} c \mathfrak{P}\left(p^{k}(p-1)+p^{k-1}\left(p-i-1, i p^{k-1}\right)\right. \\
& \times \Phi_{-1, k-1} y^{p k(p-1)}+\lambda_{k} \mathfrak{P}^{p k+1} y^{p^{k}(p-1)} \bmod \text { zero. }
\end{aligned}
$$

The second term of the right-hand side in (7.26) is obviously zero. Furthermore, we have by the hypothesis of induction, Lemma 2.3, Lemma 2.4 and $M^{\prime}(k-2) y^{p k-1 n}=0$ 


$$
\begin{aligned}
& c \mathfrak{P}\left(p^{k}(p-1)+p^{k-1}(p-i-1)\right) \Phi_{-1, k-1} y^{p k(p-1)} \\
& =c\left(\mathfrak{B}^{p^{k-1}(p-i-1)} \mathfrak{P}^{p k_{(p-1)}}\right) y^{p k-1(p+1)(p-1)} \\
& =c \mathfrak{P}^{p_{(p-1)}} c \mathfrak{P}^{p k-1(p-i-1)} y^{p k-1(p+1)(p-1)} \\
& =(-1)^{i} c \mathfrak{P}^{p^{k}(p-1)} \mathfrak{F}^{p^{k-1}(p-i-1)} y^{p^{k-1}\left(p^{2}-1\right)} \\
& =-c \mathfrak{P}^{p^{k}(p-1)} y^{p k-1(2 p-i)(p-1)} \text {. }
\end{aligned}
$$

Next, by the same device used in the proof of (7.18) we can obtain

$$
\begin{array}{rlr}
c \mathfrak{P}\left(p^{k}(p-1)+p^{k-1}(p-i-1), i p^{k-1}\right) \Phi_{-1}, k-1 & y^{p^{k}(p-1)} & \\
=c \mathfrak{P}^{i p^{k-1}} \mathfrak{P}^{p k(p-1)} y^{p^{k-1}(2 p-i)(p-1)}\left\{\begin{array}{lr}
=0 & 0<i \neq p-1 \\
=y^{p k}(p+1)(p-1) & i=p-1
\end{array}\right.
\end{array}
$$

Therefore, we have finally

$$
\Phi_{-1, k} y^{p^{k+1}(p-1)}=y^{p^{k}(p+1)(p-1)} \quad \bmod z e r o .
$$

To compute $\Phi_{-1},{ }_{k} y^{p k+1} n$ for general $n$, we can utilize the following expression of $\mathfrak{P}^{p k+1}$ in terms of secondary operations (see the proof of Theorem 5.6).

$$
\begin{gathered}
\mathfrak{P}^{p^{k+1}} \equiv c(\Delta) \Phi_{k}^{(2)}-c\left(\Delta \mathfrak{P}^{p^{k}(p-1)-1}\right) \Phi_{0, k}-c\left(\mathfrak{P}^{p^{k}(p-1}\right) \Phi_{-1, k} \\
\bmod \sum_{j<k} A \Phi_{i, j}+\sum_{\substack{j \leq i \leq 2 \\
j<k}} A \Phi_{j}(i)
\end{gathered}
$$

Thus, in a similar way as in the proof of (7.20), our theorem is proved.

\section{References}

[1] J. F. Adams, On the structure and applications of the Steenrod algebra, Comment. Math. Helv. 32 (1958), 180-214.

[2] J. F. Adams, On the non-existence of elements of Hopf invariant one, Bull. Amer. Math. Soc. 64 (1958), 279-282.

[3] J. F. Adams, On the non-existence of elements of Hopf invariant one, Ann. of Math. 72 (1960), 20-104.

[4] J. Adem, The iteration of the Steenrod squares in algebraic topology, Proc. Nat. Acad. Sci. U.S. A. 38 (1952), 720-726.

[5] J. Adem, The relations on Steenrod powers of cohomology classes, Algebraic geometry and topology, Princeton Univ. Press, 1957, 191-238.

[6] H. Cartan, Sur l'itération des opération de Steenrod, Comment. Math. Helv. 29 (1955), 40-58.

[7] A. Liulevicius, The factorization of cyclic reduced powers by secondary cohomology operations, Proc. Nat. Acad. Sci. U.S. A. 46 (1960), 978-981.

[8] J. Milnor, The Steenrod algebra and its dual, Ann. of Math. 67 (1958), 150-171.

[9] S. Mukohda, On a theorem of Toda in the Steenrod algebra, Memoirs of Fac. Sci. Kyushu Univ. Ser. A, 14 (1960), 85-97.

[10] A. Negishi, Exact sequences in the Steenrod algebra, J. of Math. Soc. 10 (1958), 71-78.

[11] Y. Nomura, On mapping sequences, Nagoya Math. J. 17 (1960), 111-145.

[12] F. P. Peterson and N. Stein, Secondary cohomology operations: two formulas, Amer. J. Math. 81 (1951), 281-305.

[13] J. P. Serre, Homologie singulière des espaces fibrés, Ann. of Math. 54 (1951), 425-505. 
[14] N. Shimada, Triviality of the $\bmod p$ Hopf invariants, Proc. Jap. Acad. 36 (1960), 68-69.

[15] N. Shimada, On stable functional cohomology operations, Proc. Jap. Acad. 36 (1960), 183-186.

[16] N. E. Steenrod, Cohomology invariants of mappings, Ann. of Math. 50 (1949), 954-988.

[17] H. Toda, p-primary components of homotopy groups 1, II, III, Memoirs of Coll. Sci. Univ. Kyoto 31 (1958), 129-142, 143-160, 191-210.

[18] T. Yamanoshita, On the mod $p$ Hopf invariant, Proc. Jap. Acad. 36 (1960), 97-98. 